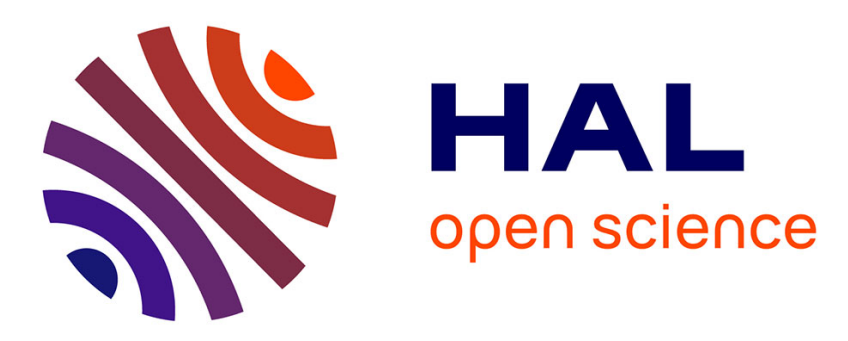

\title{
Proximal algorithms for multicomponent image recovery problems
}

\author{
Luis M. Briceno-Arias, Patrick Louis Combettes, Jean-Christophe Pesquet, \\ Nelly Pustelnik
}

\section{> To cite this version:}

Luis M. Briceno-Arias, Patrick Louis Combettes, Jean-Christophe Pesquet, Nelly Pustelnik. Proximal algorithms for multicomponent image recovery problems. Journal of Mathematical Imaging and Vision, 2011, 41 (1-2), pp.3-22. 10.1007/s10851-010-0243-1 . hal-00826810

\section{HAL Id: hal-00826810 https://hal.science/hal-00826810}

Submitted on 28 May 2013

HAL is a multi-disciplinary open access archive for the deposit and dissemination of scientific research documents, whether they are published or not. The documents may come from teaching and research institutions in France or abroad, or from public or private research centers.
L'archive ouverte pluridisciplinaire HAL, est destinée au dépôt et à la diffusion de documents scientifiques de niveau recherche, publiés ou non, émanant des établissements d'enseignement et de recherche français ou étrangers, des laboratoires publics ou privés. 


\title{
Proximal algorithms for multicomponent image recovery problems *
}

\author{
L. M. Briceño-Ariasł P. L. Combettes; J.-C. Pesquet, N. Pustelnik.
}

May 28, 2013

\begin{abstract}
In recent years, proximal splitting algorithms have been applied to various monocomponent signal and image recovery problems. In this paper, we address the case of multicomponent problems. We first provide closed form expressions for several important multicomponent proximity operators and then derive extensions of existing proximal algorithms to the multicomponent setting. These results are applied to stereoscopic image recovery, multispectral image denoising, and image decomposition into texture and geometry components.
\end{abstract}

\section{Problem statement}

In this paper, we consider signal and image recovery problems in which the ideal solution is represented by $m$ components $x_{1}, \ldots, x_{m}$ lying, respectively, in real Hilbert spaces $\mathcal{H}_{1}, \ldots, \mathcal{H}_{m}$. Such problems arise in many areas ranging from color and hyperspectral imaging to multichannel signal processing and geometry/texture image decomposition [2, 4, 5, 6, 7, 12, 23, 25, 29, 30, 40, 43, 46]. Oftentimes, multicomponent signal/image processing tasks can be formulated as variational problems of the form

$$
\underset{x_{1} \in \mathcal{H}_{1}, \ldots, x_{m} \in \mathcal{H}_{m}}{\operatorname{minimize}} \Phi\left(x_{1}, \ldots, x_{m}\right),
$$

where $\Phi$ is a convex function modeling the available information on the $m$ components, their interactions, and, possibly, the data acquisition process.

\footnotetext{
${ }^{*}$ Part of this work appeared in the conference proceedings of EUSIPCO 2010 [37]. This work was supported by the Agence Nationale de la Recherche under grants ANR-08-BLAN-0294-02 and ANR-09-EMER-004-03.

${ }^{\dagger}$ Luis M. Briceño-Arias is with the Center for Mathematical Modeling, CNRS, UMI 2807, Blanco Encalada 2120, 6th oor of. 621, Santiago de Chile, Phone: (+56 9)9 8391744 E-mail: lbriceno@math.jussieu.fr.

${ }^{\ddagger}$ Patrick. L. Combettes is with the UPMC Universite Paris 06, Laboratoire Jacques-Louis Lions, CNRS UMR 7598, 75005 Paris, France. Phone: (+33) 1442763 19, E-mail: plc@math.jussieu.fr.

§ Jean-Christophe Pesquet is with the Laboratoire d'Informatique Gaspard Monge, CNRS UMR 8049, 77454 Marne la Vallée Cedex 2, France. $\quad$ Phone: $\quad(+33) \quad 1 \quad 60 \quad 95 \quad 77$ 39, E-mail: jean-christophe.pesquet@univ-paris-est.fr.

${ }^{\top}$ N. Pustelnik is with the Laboratoire de Physique de l'ENS Lyon, CNRS UMR 5672, F69007 Lyon, France. Phone: (+33) 4727286 49, E-mail: nelly.pustelnik@ens-lyon.fr.
} 
The abstract convex minimization problem (1) is usually too generic to be solved directly and it must be formulated in a more structured fashion to be amenable to efficient numerical solution. To this end, $\Phi$ can be decomposed as a sum of $p$ functions that can be handled individually more easily. This leads to the following model, which will be the focus of the paper.

Problem 1.1 Let $\left(\mathcal{H}_{i}\right)_{1 \leq i \leq m}$ be real Hilbert spaces, and let $\left(f_{k}\right)_{1 \leq k \leq p}$ be proper lower semicontinuous convex functions from the direct Hilbert sum $\mathcal{H}_{1} \oplus \cdots \oplus \mathcal{H}_{m}$ to $\left.]-\infty,+\infty\right]$. The problem is to

$$
\underset{x_{1} \in \mathcal{H}_{1}, \ldots, x_{m} \in \mathcal{H}_{m}}{\operatorname{minimize}} \sum_{k=1}^{p} f_{k}\left(x_{1}, \ldots, x_{m}\right),
$$

under the assumption that solutions exist.

In the case of univariate $(m=1)$ signal processing problems, proximal methods have been successfully used to solve $(2)$; see $[17,19,21]$ for basic work, and [20] and the references therein for a variety of applications. It is therefore natural to ask whether these methods can be extended to the multivariate case. Initial work in this direction was carried out in [10] in the special instance when $m=2, f_{1}$ is a separable sum (i.e., $f_{1}:\left(x_{i}\right)_{1 \leq i \leq m} \mapsto \sum_{i=1}^{m} \varphi_{i}\left(x_{i}\right)$ ), and $f_{2}$ is differentiable on $\mathcal{H}_{1} \oplus \cdots \oplus \mathcal{H}_{m}$ with a Lipschitz continuous gradient (this setting also covers formulations found in $[4,5,6,21,26,27,41,42,46])$. The objective of our paper is to address the general case and to present several proximal algorithms with guaranteed convergence to a solution to Problem 1.1 under suitable assumptions.

The paper is organized as follows. In section 2, the main notation used in the paper is introduced. Proximity operators will be an essential ingredient in the multicomponent algorithms proposed in the paper. They are briefly reviewed in section 3, where we also provide new results concerning multicomponent proximity operators. In section 4, we describe proximal splitting algorithms which are pertinent for solving Problem 1.1. Finally, in section 5, we illustrate the effectiveness of the proposed algorithms in three multicomponent imaging examples.

\section{Notation}

Throughout, $\mathcal{H}, \mathcal{G}$, and $\left(\mathcal{H}_{i}\right)_{1 \leq i \leq m}$ are real Hilbert spaces. For convenience, their scalar products are all denoted by $\langle\cdot \mid \cdot\rangle$, the associated norms by $\|\cdot\|$, and their identity operators are all denoted by Id. It will be convenient to denote by $\boldsymbol{x}=\left(x_{i}\right)_{1 \leq i \leq m}$ a generic element in $\mathcal{H}_{1} \times \cdots \times \mathcal{H}_{m}$, and by $\mathcal{H}$ the direct Hilbert sum $\mathcal{H}_{1} \oplus \cdots \oplus \mathcal{H}_{m}$, i.e., the product space $\mathcal{H}_{1} \times \cdots \times \mathcal{H}_{m}$ equipped with the usual vector space structure and the scalar product

$$
(\boldsymbol{x}, \boldsymbol{y}) \mapsto \sum_{i=1}^{m}\left\langle x_{i} \mid y_{i}\right\rangle
$$

The space of bounded linear operators from $\mathcal{H}$ to $\mathcal{G}$ is denoted by $\mathcal{B}(\mathcal{H}, \mathcal{G})$. Moreover, $\Gamma_{0}(\mathcal{H})$ denotes the class of lower semicontinuous convex functions $\varphi: \mathcal{H} \rightarrow]-\infty,+\infty$ ] which are proper in the sense that

$$
\operatorname{dom} \varphi=\{x \in \mathcal{H} \mid \varphi(x)<+\infty\} \neq \varnothing
$$


Let $C$ and $D$ be nonempty convex subsets of $\mathcal{H}$. The indicator function of $C$ is

$$
\iota_{C}: x \mapsto \begin{cases}0, & \text { if } x \in C ; \\ +\infty, & \text { if } x \notin C .\end{cases}
$$

If $C$ is closed, for every $x \in \mathcal{H}$, there exists a unique point $P_{C} x \in C$ such that $\left\|x-P_{C} x\right\|=$ $\inf _{y \in C}\|x-y\| ; P_{C} x$ is called the projection of $x$ onto $C$. We say that 0 lies in the strong relative interior of $C$, in symbol, $0 \in \operatorname{sri} C$, if $\bigcup_{\lambda>0} \lambda C=\overline{\operatorname{span}} C$. In particular, if we set $C-D=$ $\{x-y \mid(x, y) \in C \times D\}$, the inclusion $0 \in \operatorname{sri}(C-D)$ holds in each of the following cases:

- $C-D$ is a closed vector subspace.

- $0 \in \operatorname{int}(C-D)$.

- $C \cap \operatorname{int} D \neq \varnothing$.

- $\mathcal{H}$ is finite dimensional and $($ ri $C) \cap($ ri $D) \neq \varnothing$, where ri $C$ denotes the relative interior of $C$, i.e., its interior relative to its affine hull.

General background on convex analysis will be found in $[8,47]$.

\section{Proximity operators}

\subsection{Definition and properties}

For a detailed account of the theory of proximity operators, see [8] and the classical paper [33].

Let $\varphi \in \Gamma_{0}(\mathcal{H})$. For every $x \in \mathcal{H}$, the function

$$
y \mapsto \varphi(y)+\frac{1}{2}\|x-y\|^{2}
$$

has a unique minimizer, which is denoted by $\operatorname{prox}_{\varphi} x$ and characterized by the variational inequality

$$
(\forall p \in \mathcal{H}) \quad p=\operatorname{prox}_{\varphi} x \quad \Leftrightarrow \quad(\forall y \in \mathcal{H}) \quad\langle y-p \mid x-p\rangle+\varphi(p) \leq \varphi(y) .
$$

The proximity operator $\operatorname{prox}_{\varphi}$ of $\varphi$ thus defined is nonexpansive, i.e.,

$$
(\forall x \in \mathcal{H})(\forall y \in \mathcal{H}) \quad\left\|\operatorname{prox}_{\varphi} x-\operatorname{prox}_{\varphi} y\right\| \leq\|x-y\| .
$$

Example 3.1 Let $C$ be a nonempty closed convex subset of $\mathcal{H}$. Then $\operatorname{prox}_{\iota_{C}}=P_{C}$. 33].

Other closed-form expressions for the proximity operators can be found in $[3,10,14,17,18,21$,

Lemma 3.2 [17, Proposition 11] Let $\psi \in \Gamma_{0}(\mathcal{G})$, let $L \in \mathcal{B}(\mathcal{H}, \mathcal{G})$, and set $\varphi=\psi \circ L$. Suppose that $L \circ L^{*}=\alpha \mathrm{Id}$, for some $\left.\alpha \in\right] 0,+\infty\left[\right.$. Then $\varphi \in \Gamma_{0}(\mathcal{H})$ and

$$
\operatorname{prox}_{\varphi}=\operatorname{Id}+\frac{1}{\alpha} L^{*} \circ\left(\operatorname{prox}_{\alpha \psi}-\mathrm{Id}\right) \circ L \text {. }
$$




\subsection{Multicomponent proximity operators}

The computation of proximity operators in the Hilbert direct sum $\mathcal{H}$ will play a fundamental role in the next sections. Below, we provide some important situations in which this computation is explicit.

Proposition 3.3 Suppose that, for every $i \in\{1, \ldots, m\},\left(e_{i, k}\right)_{k \in \mathbb{K}}$ is an orthonormal basis of $\mathcal{H}_{i}$. Furthermore, let $\left(\phi_{k}\right)_{k \in \mathbb{K}}$ be functions in $\Gamma_{0}\left(\mathbb{R}^{m}\right)$ and suppose that one of the following holds.

(i) For every $i \in\{1, \ldots, m\}, \mathcal{H}_{i}$ is infinite dimensional and, for every $k \in \mathbb{K}, \phi_{k} \geq \phi_{k}(\mathbf{0})=0$.

(ii) For every $i \in\{1, \ldots, m\}, \mathcal{H}_{i}$ is finite dimensional.

Set

$$
\begin{aligned}
f: \mathcal{H} & \rightarrow]-\infty,+\infty] \\
\boldsymbol{x} & \mapsto \sum_{k \in \mathbb{K}} \phi_{k}\left(\left\langle x_{1} \mid e_{1, k}\right\rangle, \ldots,\left\langle x_{m} \mid e_{m, k}\right\rangle\right) .
\end{aligned}
$$

Then $f \in \Gamma_{0}(\mathcal{H})$ and, for every $\boldsymbol{x} \in \mathcal{H}$,

$$
\operatorname{prox}_{f} \boldsymbol{x}=\left(\sum_{k \in \mathbb{K}} \pi_{1, k} e_{1, k}, \ldots, \sum_{k \in \mathbb{K}} \pi_{m, k} e_{m, k}\right),
$$

where

$$
(\forall k \in \mathbb{K}) \quad\left(\pi_{1, k}, \ldots, \pi_{m, k}\right)=\operatorname{prox}_{\phi_{k}}\left(\left\langle x_{1} \mid e_{1, k}\right\rangle, \ldots,\left\langle x_{m} \mid e_{m, k}\right\rangle\right) .
$$

Proof 3.4 Let us set, for every $k \in \mathbb{K}$,

$$
\begin{aligned}
\psi_{k}: \mathcal{H} & \rightarrow]-\infty,+\infty] \\
\boldsymbol{x} & \mapsto \phi_{k}\left(\left\langle x_{1} \mid e_{1, k}\right\rangle, \ldots,\left\langle x_{m} \mid e_{m, k}\right\rangle\right) .
\end{aligned}
$$

Then our assumptions imply that the functions $\left(\psi_{k}\right)_{k \in \mathbb{K}}$ are in $\Gamma_{0}(\mathcal{H})$. Under assumption (i), assuming without loss of generality that $\mathbb{K}=\mathbb{N}$, we can write $f=\sup _{K \in \mathbb{K}} \sum_{k=0}^{K} \psi_{k}$. Since lower semicontinuity and convexity are preserved under finite sums and taking suprema, it follows that $f$ is lower semicontinuous and convex. In addition, since $f(\mathbf{0})=0$, we obtain $f \in \Gamma_{0}(\mathcal{H})$. On the other hand, under assumption (ii), the sum in (10) is finite and our assumptions imply at once that $f \in \Gamma_{0}(\mathcal{H})$.

Now let $\boldsymbol{x} \in \mathcal{H}$ and denote the Euclidean norm on $\mathbb{R}^{m}$ by $|\cdot|$. Set

$$
(\forall i \in\{1, \ldots, m\})(\forall k \in \mathbb{K}) \quad \xi_{i, k}=\left\langle x_{i} \mid e_{i, k}\right\rangle .
$$

Moreover, for every $i \in\{1, \ldots, m\}$, let $y_{i} \in \mathcal{H}_{i}$ and set $\left(\eta_{i, k}\right)_{k \in \mathbb{K}}=\left(\left\langle y_{i} \mid e_{i, k}\right\rangle\right)_{k \in \mathbb{K}}$. We derive from (12) and (7) that, for every $k \in \mathbb{K}$,

$$
\sum_{i=1}^{m}\left(\eta_{i, k}-\pi_{i, k}\right)\left(\xi_{i, k}-\pi_{i, k}\right)+\phi_{k}\left(\pi_{1, k}, \ldots, \pi_{m, k}\right) \leq \phi_{k}\left(\eta_{1, k}, \ldots, \eta_{m, k}\right) .
$$


Let us first assume that (i) holds. For every $k \in \mathbb{K}$ observe that, since $\mathbf{0}$ is a minimizer of $\phi_{k},(7)$ yields $\operatorname{prox}_{\phi_{k}} \mathbf{0}=\mathbf{0}$. Hence, using (12), (14), (8), and Parseval's identity, we obtain

$$
\begin{aligned}
\sum_{k \in \mathbb{K}} \sum_{i=1}^{m}\left|\pi_{i, k}\right|^{2} & =\sum_{k \in \mathbb{K}}\left|\left(\pi_{1, k}, \ldots, \pi_{m, k}\right)\right|^{2} \\
& =\sum_{k \in \mathbb{K}}\left|\operatorname{prox}_{\phi_{k}}\left(\xi_{1, k}, \ldots, \xi_{m, k}\right)\right|^{2} \\
& =\sum_{k \in \mathbb{K}}\left|\operatorname{prox}_{\phi_{k}}\left(\xi_{1, k}, \ldots, \xi_{m, k}\right)-\operatorname{prox}_{\phi_{k}} \mathbf{0}\right|^{2} \\
& \leq \sum_{k \in \mathbb{K}}\left|\left(\xi_{1, k}, \ldots, \xi_{m, k}\right)-\mathbf{0}\right|^{2} \\
& =\sum_{k \in \mathbb{K}}\left|\left(\xi_{1, k}, \ldots, \xi_{m, k}\right)\right|^{2} \\
& =\sum_{k \in \mathbb{K}} \sum_{i=1}^{m}\left|\xi_{i, k}\right|^{2} \\
& =\sum_{i=1}^{m}\left\|x_{i}\right\|^{2} .
\end{aligned}
$$

Therefore, $(\forall i \in\{1, \ldots, m\}) \sum_{k \in \mathbb{K}}\left|\pi_{i, k}\right|^{2}<+\infty$. Consequently, we can define

$$
(\forall i \in\{1, \ldots, m\}) \quad z_{i}=\sum_{k \in \mathbb{K}} \pi_{i, k} e_{i, k},
$$

which is equally well defined under assumption (ii) as $\mathbb{K}$ is then finite. It remains to show that $\left(z_{i}\right)_{1 \leq i \leq m}=\operatorname{prox}_{f}\left(x_{1}, \ldots, x_{m}\right)$. Summing over $k$ in (15) yields

$$
\sum_{k \in \mathbb{K}} \sum_{i=1}^{m}\left(\eta_{i, k}-\pi_{i, k}\right)\left(\xi_{i, k}-\pi_{i, k}\right)+\sum_{k \in \mathbb{K}} \phi_{k}\left(\pi_{1, k}, \ldots, \pi_{m, k}\right) \leq \sum_{k \in \mathbb{K}} \phi_{k}\left(\eta_{1, k}, \ldots, \eta_{m, k}\right)
$$

and, therefore,

$$
\sum_{i=1}^{m}\left\langle y_{i}-z_{i} \mid x_{i}-z_{i}\right\rangle+g\left(z_{1}, \ldots, z_{m}\right) \leq g\left(y_{1}, \ldots, y_{m}\right) .
$$

In view of $(7)$, the proof is complete.

Proposition 3.5 For every $j \in\{1, \ldots, q\}$, let $\mathcal{G}_{j}$ be a real Hilbert space, let $\varphi_{j} \in \Gamma_{0}\left(\mathcal{G}_{j}\right)$, and, for every $i \in\{1, \ldots, m\}$, let $L_{j, i} \in \mathcal{B}\left(\mathcal{H}_{i}, \mathcal{G}_{j}\right)$. Set

$$
\begin{aligned}
f: \mathcal{H} & \rightarrow]-\infty,+\infty] \\
\boldsymbol{x} & \mapsto \sum_{j=1}^{q} \varphi_{j}\left(\sum_{i=1}^{m} L_{j, i} x_{i}\right)
\end{aligned}
$$

and suppose that, for every $j \in\{1, \ldots, q\}$, there exists $\left.\alpha_{j} \in\right] 0,+\infty[$ such that

$$
(\forall k \in\{1, \ldots, q\}) \quad \sum_{i=1}^{m} L_{j, i} \circ L_{k, i}^{*}= \begin{cases}\alpha_{j} \mathrm{Id}, & \text { if } j=k ; \\ 0, & \text { otherwise. }\end{cases}
$$


Then $f \in \Gamma_{0}(\mathcal{H})$ and, for every $\boldsymbol{x} \in \mathcal{H}$,

$$
\operatorname{prox}_{f} \boldsymbol{x}=\left(p_{1}, \ldots, p_{m}\right)
$$

where, for every $i \in\{1, \ldots, m\}$,

$$
p_{i}=x_{i}+\sum_{j=1}^{q} \alpha_{j}^{-1} L_{j, i}^{*} \operatorname{prox}_{\alpha_{j} \varphi_{j}}\left(\sum_{k=1}^{m} L_{j, k} x_{k}\right)-\sum_{j=1}^{q} \alpha_{j}^{-1} L_{j, i}^{*} \sum_{k=1}^{m} L_{j, k} x_{k} .
$$

Proof 3.6 Let us denote by $\mathcal{G}$ the product space $\mathcal{G}_{1} \times \cdots \times \mathcal{G}_{q}$ equipped with the usual vector space structure and the scalar product

$$
(\boldsymbol{y}, \boldsymbol{z}) \mapsto \sum_{j=1}^{q} \alpha_{j}^{-1}\left\langle y_{j} \mid z_{j}\right\rangle .
$$

We can write $f=g \circ \boldsymbol{L}$, where

$$
\begin{aligned}
g: \mathcal{G} & \rightarrow]-\infty,+\infty] \\
\boldsymbol{y} & \mapsto \sum_{j=1}^{q} \varphi_{j}\left(y_{j}\right)
\end{aligned}
$$

and $\boldsymbol{L} \in \mathcal{B}(\mathcal{H}, \mathcal{G})$ is defined by

$$
\begin{aligned}
\boldsymbol{L}: \mathcal{H} & \rightarrow \mathcal{G} \\
\boldsymbol{x} & \mapsto\left(\sum_{i=1}^{m} L_{1, i} x_{i}, \ldots, \sum_{i=1}^{m} L_{q, i} x_{i}\right) .
\end{aligned}
$$

It follows from (24) that, for every $(\boldsymbol{x}, \boldsymbol{y}) \in \mathcal{H} \times \mathcal{G}$,

$$
\begin{aligned}
\langle\boldsymbol{L} \boldsymbol{x} \mid \boldsymbol{y}\rangle & =\sum_{j=1}^{q} \alpha_{j}^{-1}\left\langle\sum_{i=1}^{m} L_{j, i} x_{i} \mid y_{j}\right\rangle \\
& =\sum_{j=1}^{q} \alpha_{j}^{-1} \sum_{i=1}^{m}\left\langle x_{i} \mid L_{j, i}^{*} y_{j}\right\rangle \\
& =\sum_{i=1}^{m}\left\langle x_{i} \mid \sum_{j=1}^{q} \alpha_{j}^{-1} L_{j, i}^{*} y_{j}\right\rangle,
\end{aligned}
$$

from which we deduce that the adjoint of $\boldsymbol{L}$ is

$$
\begin{aligned}
\boldsymbol{L}^{*}: \mathcal{G} & \rightarrow \mathcal{H} \\
\boldsymbol{y} & \mapsto\left(\sum_{j=1}^{q} \alpha_{j}^{-1} L_{j, 1}^{*} y_{j}, \ldots, \sum_{j=1}^{q} \alpha_{j}^{-1} L_{j, m}^{*} y_{j}\right) .
\end{aligned}
$$

We then get from (21) that $\boldsymbol{L} \circ \boldsymbol{L}^{*}=\mathrm{Id}$. Hence, Lemma 3.2 implies that $f=g \circ \boldsymbol{L} \in \Gamma_{0}(\mathcal{H})$ and that

$$
\operatorname{prox}_{g \circ \boldsymbol{L}}=\operatorname{Id}+\boldsymbol{L}^{*} \circ\left(\operatorname{prox}_{g}-\mathrm{Id}\right) \circ \boldsymbol{L} .
$$


In addition, it follows from (25) and (24) that, for every $\boldsymbol{y} \in \mathcal{G}$,

$$
\operatorname{prox}_{g} \boldsymbol{y}=\left(\operatorname{prox}_{\alpha_{1} \varphi_{1}} y_{1}, \ldots, \operatorname{prox}_{\alpha_{q} \varphi_{q}} y_{q}\right) \text {. }
$$

Altogether, (26), (28), (29), and (30) yield (22)-(23).

Corollary 3.7 Let $\varphi \in \Gamma_{0}(\mathcal{G})$ and, for every $i \in\{1, \ldots, m\}$, let $L_{i} \in \mathcal{B}\left(\mathcal{H}_{i}, \mathcal{G}\right)$. Set

$$
\begin{aligned}
f: \mathcal{H} & \rightarrow]-\infty,+\infty] \\
\boldsymbol{x} & \mapsto \varphi\left(\sum_{i=1}^{m} L_{i} x_{i}\right)
\end{aligned}
$$

and suppose that there exists $\alpha \in] 0,+\infty[$ such that

$$
\sum_{i=1}^{m} L_{i} \circ L_{i}^{*}=\alpha \mathrm{Id} .
$$

Then $f \in \Gamma_{0}(\mathcal{H})$ and, for every $\boldsymbol{x} \in \mathcal{H}, \operatorname{prox}_{f} \boldsymbol{x}=\left(p_{1}, \ldots, p_{m}\right)$ where, for every $i \in\{1, \ldots, m\}$,

$$
p_{i}=x_{i}+\alpha^{-1} L_{i}^{*} \operatorname{prox}_{\alpha \varphi}\left(\sum_{k=1}^{m} L_{k} x_{k}\right)-\alpha^{-1} L_{i}^{*} \sum_{k=1}^{m} L_{k} x_{k} .
$$

Proof 3.8 Set $q=1$ in Proposition 3.5.

Proposition 3.9 Suppose that $\mathcal{G}$ has finite dimension $K$, let $\left(\phi_{k}\right)_{1 \leq k \leq K}$ be functions in $\Gamma_{0}(\mathbb{R})$, and let $\left(e_{k}\right)_{1 \leq k \leq K}$ be an orthonormal basis of $\mathcal{G}$. For every $i \in\{1, \ldots, m\}$, let $L_{i} \in \mathcal{B}\left(\mathcal{H}_{i}, \mathcal{G}\right)$ and suppose that there exists $\left.\left\{\alpha_{k}\right\}_{1 \leq k \leq K} \subset\right] 0,+\infty[$ such that

$$
(\forall y \in \mathcal{G}) \quad \sum_{i=1}^{m} L_{i} L_{i}^{*} y=\sum_{k=1}^{K} \alpha_{k}\left\langle y \mid e_{k}\right\rangle e_{k} .
$$

Set

$$
f: \mathcal{H} \rightarrow]-\infty,+\infty]: \boldsymbol{x} \mapsto \sum_{k=1}^{K} \phi_{k}\left(\left\langle\sum_{j=1}^{m} L_{j} x_{j} \mid e_{k}\right\rangle\right)
$$

and, for every $k \in\{1 \ldots, K\}$,

$$
\pi_{k}=\frac{1}{\alpha_{k}} \operatorname{prox}_{\alpha_{k} \phi_{k}}\left(\left\langle\sum_{j=1}^{m} L_{j} x_{j} \mid e_{k}\right\rangle\right) .
$$

Then $f \in \Gamma_{0}(\mathcal{H})$ and, for every $\boldsymbol{x} \in \mathcal{H}, \operatorname{prox}_{f} \boldsymbol{x}=\left(p_{i}\right)_{1 \leq i \leq m}$ where, for every $i \in\{1, \ldots, m\}$,

$$
p_{i}=x_{i}+L_{i}^{*} \sum_{k=1}^{K}\left(\pi_{k}-\frac{1}{\alpha_{k}} \sum_{j=1}^{m}\left\langle L_{j} x_{j} \mid e_{k}\right\rangle\right) e_{k} .
$$


Proof 3.10 For every $j \in\{1, \ldots, K\}$, set $\mathcal{G}_{j}=\mathbb{R}, \varphi_{j}=\phi_{j}$, and

$$
(\forall i \in\{1, \ldots, m\}) L_{j, i}: \mathcal{H}_{i} \rightarrow \mathcal{G}_{j}: x \mapsto\left\langle L_{i} x \mid e_{j}\right\rangle,
$$

hence

$$
L_{j, i}^{*}: \mathcal{G}_{j} \rightarrow \mathcal{H}_{i}: \xi \mapsto \xi L_{i}^{*} e_{j}
$$

Thus, for every $j$ and $k$ in $\{1, \ldots, K\}$ and every $\xi \in \mathbb{R}$, we derive from (34) that

$$
\begin{aligned}
\sum_{i=1}^{m} L_{j, i} L_{k, i}^{*} \xi & =\sum_{i=1}^{m} L_{j, i} \xi L_{i}^{*} e_{k} \\
& =\xi \sum_{i=1}^{m}\left\langle\left(L_{i} L_{i}^{*}\right) e_{k} \mid e_{j}\right\rangle \\
& =\xi\left\langle\sum_{l=1}^{K} \alpha_{l}\left\langle e_{k} \mid e_{l}\right\rangle e_{l} \mid e_{j}\right\rangle \\
& =\xi \sum_{l=1}^{K} \alpha_{l}\left\langle e_{k} \mid e_{l}\right\rangle\left\langle e_{l} \mid e_{j}\right\rangle .
\end{aligned}
$$

Therefore, for every $j \in\{1, \ldots, K\}$, (21) is satisfied. In turn, Proposition 3.5 with $q=K$ guarantees that $f \in \Gamma_{0}(\mathcal{H})$, and $(23)$ reduces to $(37)$.

\section{Multicomponent proximal algorithms}

We present several algorithms for solving Problem 1.1 under various assumptions on the functions involved. Most of these algorithms are tolerant to errors in the computation of proximal points and gradients. To quantify the amount of error which is tolerated, it will be convenient to use the following notation: given two sequences $\left(\boldsymbol{x}_{n}\right)_{n \in \mathbb{N}}$ and $\left(\boldsymbol{y}_{n}\right)_{n \in \mathbb{N}}$ in $\mathcal{H}$,

$$
\left[(\forall n \in \mathbb{N}) \boldsymbol{x}_{n} \approx \boldsymbol{y}_{n}\right] \Leftrightarrow \sum_{n \in \mathbb{N}}\left\|\boldsymbol{x}_{n}-\boldsymbol{y}_{n}\right\|<+\infty .
$$

\subsection{Forward-backward splitting}

Problem 4.1 In Problem 1.1, suppose that $p=2$ and that $f_{2}$ is differentiable on $\mathcal{H}$ with a $\beta$-Lipschitz continuous gradient for some $\beta \in] 0,+\infty[$. Hence, the problem is to

$$
\underset{x_{1} \in \mathcal{H}_{1}, \ldots, x_{m} \in \mathcal{H}_{m}}{\operatorname{minimize}} f_{1}\left(x_{1}, \ldots, x_{m}\right)+f_{2}\left(x_{1}, \ldots, x_{m}\right),
$$

under the assumption that solutions exist.

The particular case when $f_{1}$ is a separable sum and $f_{2}$ involves a linear mixture of the variables was investigated in [10]. The following result addresses the general case; it implicitly assumes that the proximity operator of $f_{1}$ can be computed to within a quantifiable error. 
Theorem 4.1 Let $\left(x_{1, n}\right)_{n \in \mathbb{N}}, \ldots,\left(x_{m, n}\right)_{n \in \mathbb{N}}$ be sequences generated by the following routine.

$$
\begin{aligned}
& \text { Initialization } \\
& \qquad \begin{array}{l}
\varepsilon \in] 0, \min \{1,1 / \beta\}[ \\
\text { For } i=1, \ldots, m \\
\left\lfloor x_{i, 0} \in \mathcal{H}_{i}\right.
\end{array}
\end{aligned}
$$

For $n=0,1, \ldots$

$$
\mid \begin{aligned}
& \left(y_{i, n}\right)_{1 \leq i \leq m} \approx \nabla f_{2}\left(x_{i, n}\right)_{1 \leq i \leq m} \\
& \gamma_{n} \in[\varepsilon,(2 / \beta)-\varepsilon] \\
& \left(u_{i, n}\right)_{1 \leq i \leq m} \approx \operatorname{prox}_{\gamma_{n} f_{1}}\left(x_{i, n}-\gamma_{n} y_{i, n}\right)_{1 \leq i \leq m} \\
& \lambda_{n} \in[\varepsilon, 1] \\
& \text { For } i=1, \ldots, m \\
& \left\lfloor x_{i, n+1}=x_{i, n}+\lambda_{n}\left(u_{i, n}-x_{i, n}\right)\right.
\end{aligned}
$$

Then, for every $i \in\{1, \ldots, m\},\left(x_{i, n}\right)_{n \in \mathbb{N}}$ converges weakly to a point $x_{i} \in \mathcal{H}_{i}$. Moreover, $\left(x_{i}\right)_{1 \leq i \leq m}$ is a solution to Problem 4.1.

Proof 4.2 Apply [21, Theorem 3.4(i)] in $\mathcal{H}$ and use (3).

\section{Remark 4.3}

(i) Multicomponent version of variants of the above forward-backward algorithm such as the Nesterov-like first-order methods [9, 34, 45] can be obtained by similar reformulations in $\mathcal{H}$. However, for these methods, convergence of the iterates $\left(\left(x_{i, n}\right)_{1 \leq i \leq m}\right)_{n \in \mathbb{N}}$ to a solution to Problem 4.1 is not guaranteed, even in a finite-dimensional setting.

(ii) Strong convergence conditions in Theorem 4.1 can be derived from [21, Theorem 3.4(iv)].

\subsection{Douglas-Rachford splitting}

In this section, we relax the assumption of smoothness on $f_{2}$ and assume that its proximity operator is implementable to within a quantifiable error.

Problem 4.2 In Problem 1.1, suppose that $p=2$ and that

$$
\mathbf{0} \in \operatorname{sri}\left(\operatorname{dom} f_{1}-\operatorname{dom} f_{2}\right) .
$$

Hence, the problem is to

$$
\underset{x_{1} \in \mathcal{H}_{1}, \ldots, x_{m} \in \mathcal{H}_{m}}{\operatorname{minimize}} f_{1}\left(x_{1}, \ldots, x_{m}\right)+f_{2}\left(x_{1}, \ldots, x_{m}\right),
$$

under the assumption that solutions exist. 
Theorem 4.4 Let $\left(x_{1, n}\right)_{n \in \mathbb{N}}, \ldots,\left(x_{m, n}\right)_{n \in \mathbb{N}}$ be sequences generated by the following routine.

Initialization

$$
\left\lfloor\begin{array}{l}
\varepsilon \in] 0,1[ \\
\gamma \in] 0,+\infty[ \\
\text { For } i=1, \ldots, m \\
\left\lfloor x_{i, 0} \in \mathcal{H}_{i}\right.
\end{array}\right.
$$

For $n=0,1, \ldots$

$$
\mid \begin{aligned}
& \left(y_{i, n}\right)_{1 \leq i \leq m} \approx \operatorname{prox}_{\gamma f_{2}}\left(x_{i, n}\right)_{1 \leq i \leq m} \\
& \left(u_{i, n}\right)_{1 \leq i \leq m} \approx \operatorname{prox}_{\gamma f_{1}}\left(2 y_{i, n}-x_{i, n}\right)_{1 \leq i \leq m} \\
& \lambda_{n} \in[\varepsilon, 2-\varepsilon] \\
& \text { For } i=1, \ldots, m \\
& \left\lfloor x_{i, n+1}=x_{i, n}+\lambda_{n}\left(u_{i, n}-y_{i, n}\right) .\right.
\end{aligned}
$$

Then, for every $i \in\{1, \ldots, m\},\left(x_{i, n}\right)_{n \in \mathbb{N}}$ converges weakly to a point $x_{i} \in \mathcal{H}_{i}$. Moreover, $\operatorname{prox}_{\gamma f_{2}}\left(x_{1}, \ldots, x_{m}\right)$ is a solution to Problem 4.2. Furthermore, if $\mathcal{H}$ is finite dimensional, the sequence $\left(\left(y_{i, n}\right)_{1 \leq i \leq m}\right)_{n \in \mathbb{N}}$ converges to $\operatorname{prox}_{\gamma f_{2}}\left(x_{1}, \ldots, x_{m}\right)$.

Proof 4.5 Apply [17, Theorem 20] in $\mathcal{H}$ and use (3).

\section{Remark 4.6}

(i) Strong convergence conditions in Theorem 4.4 can be derived from [16, Theorem 2.1(ii)].

(ii) If $\mathcal{H}$ is finite dimensional, the qualification condition (43) reduces to

$$
\left(\operatorname{ridom} f_{1}\right) \cap\left(\operatorname{ridom} f_{2}\right) \neq \varnothing .
$$

\subsection{Parallel proximal algorithm (PPXA)}

The algorithm presented in this section aims at solving Problem 1.1 under minimal technical assumptions. Its cost of implementation depends on the ease of (approximate) computation of the individual proximity operators.

Problem 4.3 In Problem 1.1, suppose that

$$
\mathbf{0} \in \operatorname{sri}\left(\boldsymbol{D}-\operatorname{dom} f_{1} \times \cdots \times \operatorname{dom} f_{p}\right)
$$

where $\boldsymbol{D}=\{(\boldsymbol{x}, \ldots, \boldsymbol{x}) \mid \boldsymbol{x} \in \mathcal{H}\}$. The problem is to

$$
\underset{x_{1} \in \mathcal{H}_{1}, \ldots, x_{m} \in \mathcal{H}_{m}}{\operatorname{minimize}} \sum_{k=1}^{p} f_{k}\left(x_{1}, \ldots, x_{m}\right),
$$

under the assumption that solutions exist. 
In [1], a particular instance of Problem 4.3 in finite dimensional spaces is considered. It is approached via the alternating direction method of multipliers. The algorithm used below is an application of the PPXA algorithm proposed in [19] that allows us to address the general case.

Theorem 4.7 Let $\left(x_{1, n}\right)_{n \in \mathbb{N}}, \ldots,\left(x_{m, n}\right)_{n \in \mathbb{N}}$ be sequences generated by the following routine.

$$
\begin{aligned}
& \text { Initialization } \\
& \qquad \begin{array}{l}
\varepsilon \in] 0,1[ \\
\gamma \in] 0,+\infty[ \\
\left.\left.\left\{\omega_{k}\right\}_{1 \leq k \leq p} \subset\right] 0,1\right] \text { and } \sum_{k=1}^{p} \omega_{k}=1 \\
\text { For } i=1, \ldots, m \\
{\left[\begin{array}{l}
\text { For } k=1, \ldots, p \\
\left\lfloor y_{i, k, 0} \in \mathcal{H}_{i}\right.
\end{array}\right.} \\
\text { For } n=0,1, \ldots \\
x_{i, 0}=\sum_{k=1}^{p} \omega_{k} y_{i, k, 0}
\end{array} \\
& \qquad \begin{array}{l}
\text { For } k=1, \ldots, p \\
\left\lfloor\left(u_{i, k, n}\right)_{1 \leq i \leq m} \approx \operatorname{prox}_{\gamma f_{k} / \omega_{k}}\left(y_{i, k, n}\right)_{1 \leq i \leq m}\right. \\
\text { For } i=1, \ldots, m \\
\left\lfloor\begin{array}{l}
p \\
s_{i, n}=\sum_{k=1} \omega_{k} u_{i, k, n} \\
\lambda_{n} \in[\varepsilon, 2-\varepsilon]
\end{array}\right.
\end{array}
\end{aligned}
$$

Then, for every $i \in\{1, \ldots, m\},\left(x_{i, n}\right)_{n \in \mathbb{N}}$ converges weakly to a point $x_{i} \in \mathcal{H}_{i}$. Moreover, $\left(x_{1}, \ldots, x_{m}\right)$ is a solution to Problem 4.3.

Proof 4.8 Apply [19, Theorem 3.4] in $\mathcal{H}$ and use (3).

Remark 4.9 Suppose that $\mathcal{H}$ is finite dimensional and that

$$
\bigcap_{k=1}^{p} \operatorname{ridom} f_{k} \neq \varnothing .
$$

Then it follows from [19, Proposition 3.6(vi)] that the qualification condition (46) is satisfied.

\subsection{Dykstra-like splitting}

We consider instances of Problem 1.1 in which $f_{p}$ is a simple quadratic function. 
Problem 4.4 In Problem 1.1, suppose that $p \geq 3$, that

$$
\bigcap_{k=1}^{p-1} \operatorname{dom} f_{k} \neq \varnothing
$$

and that $f_{p}: \boldsymbol{x} \mapsto(p-1) \sum_{i=1}^{m}\left\|x_{i}-z_{i}\right\|^{2} / 2$, where $\boldsymbol{z} \in \mathcal{H}$. Hence, the problem is to

$$
\underset{x_{1} \in \mathcal{H}_{1}, \ldots, x_{m} \in \mathcal{H}_{m}}{\operatorname{minimize}} \sum_{k=1}^{p-1} f_{k}\left(x_{1}, \ldots, x_{m}\right)+\frac{p-1}{2} \sum_{i=1}^{m}\left\|x_{i}-z_{i}\right\|^{2} .
$$

Set $f=\sum_{k=1}^{p-1} f_{k} /(p-1)$. Then it follows from (49) that $f \in \Gamma_{0}(\mathcal{H})$. Hence, in view of (3), Problem 4.4 admits a unique solution, namely $\operatorname{prox}_{f} \boldsymbol{z}$.

Theorem 4.10 Let $\left(x_{1, n}\right)_{n \in \mathbb{N}}, \ldots,\left(x_{m, n}\right)_{n \in \mathbb{N}}$ be sequences generated by the following routine.

Initialization

$$
\begin{aligned}
& \left\lfloor\begin{array}{l}
\text { For } i=1, \ldots, m \\
\left\lfloor\begin{array}{l}
x_{i, 0}=z_{i} \\
\text { For } k=1, \ldots, p-1 \\
\left\lfloor y_{i, k, 0}=x_{i, 0}\right.
\end{array}\right. \\
\text { For } n=0,1, \ldots
\end{array}\right. \\
& \qquad \begin{array}{l}
\text { For } k=1, \ldots, p-1 \\
\left\lfloor\begin{array}{l}
\left(u_{i, k, n}\right) \\
1 \leq i \leq m
\end{array}=\operatorname{prox}_{f_{k}}\left(y_{i, k, n}\right)_{1 \leq i \leq m}\right. \\
\text { For } i=1, \ldots, m
\end{array} \mid \begin{array}{l}
x_{i, n+1}=\frac{1}{p-1} \sum_{k=1}^{p-1} u_{i, k, n} \\
\text { For } k=1, \ldots, p-1 \\
\left\lfloor y_{i, k, n+1}=x_{i, n+1}+y_{i, k, n}-u_{i, k, n} .\right.
\end{array}
\end{aligned}
$$

Then, for every $i \in\{1, \ldots, m\},\left(x_{i, n}\right)_{n \in \mathbb{N}}$ converges strongly to a point $x_{i} \in \mathcal{H}_{i}$. Moreover, $\left(x_{1}, \ldots, x_{m}\right)$ is a solution to Problem 4.4 .

Proof 4.11 Apply [16, Theorem 4.2] in $\mathcal{H}$ and use (3).

Remark 4.12 Suppose that (49) is replaced by the stronger condition (46) (applied to the functions $\left.\left(f_{k}\right)_{1 \leq k \leq p-1}\right)$. Then it follows from [16, Theorem 3.3] that the conclusion of the above theorem remains valid if the proximity operators are implemented approximately in (51).

\section{Applications to image decomposition and recovery}

In this section, we apply the algorithms proposed in Section 4 to stereoscopic image restoration, multispectral imaging, and image decomposition. 


\subsection{Stereoscopic image restoration}

\subsubsection{Problem formulation}

We consider the problem of restoring a pair of $N$-pixel stereoscopic images $\bar{x}_{1} \in \mathbb{R}^{N}$ and $\bar{x}_{2} \in \mathbb{R}^{N}$, which correspond to the left and the right views of the same scene. For a given value of the disparity field, the disparity compensation process between the two images is modeled as

$$
\bar{x}_{1}=D \bar{x}_{2}+v,
$$

where the matrix $D$ is in $\mathbb{R}^{N \times N}$ [39] and where $v$ stands for modeling errors. The observations consist of degraded versions

$$
z_{1}=L_{1} \bar{x}_{1}+w_{1} \quad \text { and } \quad z_{2}=L_{2} \bar{x}_{2}+w_{2}
$$

of $\bar{x}_{1}$ and $\bar{x}_{2}$, respectively, where the matrices $L_{1} \in \mathbb{R}^{N \times N}$ and $L_{2} \in \mathbb{R}^{N \times N}$ model the data acquisition process, and where $w_{1}$ and $w_{2}$ are mutually independent Gaussian noise vectors with independent components which are $\mathcal{N}\left(0, \sigma_{1}^{2}\right)$ - and $\mathcal{N}\left(0, \sigma_{2}^{2}\right)$-distributed, respectively. In addition, it is assumed that the decompositions of $\bar{x}_{1}$ and $\bar{x}_{2}$ in orthonormal bases $\left(e_{1, k}\right)_{1 \leq k \leq N}$ and $\left(e_{2, k}\right)_{1 \leq k \leq N}$, respectively, of $\mathbb{R}^{N}$ are sparse. For every $k \in\{1, \ldots, N\}$, functions $\phi_{1, k} \in \Gamma_{0}(\mathbb{R})$ and $\phi_{2, k} \in$ $\Gamma_{0}(\mathbb{R})$ are used to promote the sparsity of the decompositions $[18,22]$. The following variational formulation is consistent with the above hypotheses and models.

Problem 5.1 Let $\vartheta \in[0,+\infty[$. The objective is to

$$
\begin{aligned}
\underset{x_{1} \in \mathbb{R}^{N}, x_{2} \in \mathbb{R}^{N}}{\operatorname{minimize}} \sum_{k=1}^{N} \phi_{1, k}\left(\left\langle x_{1} \mid e_{1, k}\right\rangle\right) & +\sum_{k=1}^{N} \phi_{2, k}\left(\left\langle x_{2} \mid e_{2, k}\right\rangle\right) \\
& +\frac{1}{2 \sigma_{1}^{2}}\left\|L_{1} x_{1}-z_{1}\right\|^{2}+\frac{1}{2 \sigma_{2}^{2}}\left\|L_{2} x_{2}-z_{2}\right\|^{2}+\frac{\vartheta}{2}\left\|x_{1}-D x_{2}\right\|^{2} .
\end{aligned}
$$

We can formulate Problem 5.1 as an instance of Problem 1.1 with $\mathcal{H}_{1}=\mathcal{H}_{2}=\mathbb{R}^{N}$ and $m=2$ functions, namely

$$
f_{1}:\left(x_{1}, x_{2}\right) \mapsto \sum_{k=1}^{N} \phi_{1, k}\left(\left\langle x_{1} \mid e_{1, k}\right\rangle\right)+\sum_{k=1}^{N} \phi_{2, k}\left(\left\langle x_{2} \mid e_{2, k}\right\rangle\right)
$$

and

$$
f_{2}:\left(x_{1}, x_{2}\right) \mapsto \frac{1}{2 \sigma_{1}^{2}}\left\|L_{1} x_{1}-z_{1}\right\|^{2}+\frac{1}{2 \sigma_{2}^{2}}\left\|L_{2} x_{2}-z_{2}\right\|^{2}+\frac{\vartheta}{2}\left\|x_{1}-D x_{2}\right\|^{2} .
$$

Proposition 5.1 Let $x_{1}$ and $x_{2}$ be arbitrary vectors in $\mathbb{R}^{N}$. Then $f_{2}$ is differentiable at $\left(x_{1}, x_{2}\right)$ and

$$
\nabla f_{2}\left(x_{1}, x_{2}\right)=\left(\frac{1}{\sigma_{1}^{2}} L_{1}^{\top}\left(L_{1} x_{1}-z_{1}\right)+\vartheta\left(x_{1}-D x_{2}\right), \frac{1}{\sigma_{2}^{2}} L_{2}^{\top}\left(L_{2} x_{2}-z_{2}\right)+\vartheta D^{\top}\left(D x_{2}-x_{1}\right)\right) .
$$

Moreover, $\nabla f_{2}$ is $\beta$-Lipschitz continuous, with

$$
\beta=\max \left\{\sigma_{1}^{-2}\left\|L_{1}\right\|^{2}, \sigma_{2}^{-2}\left\|L_{2}\right\|^{2}\right\}+\vartheta\left(1+\|D\|^{2}\right) .
$$




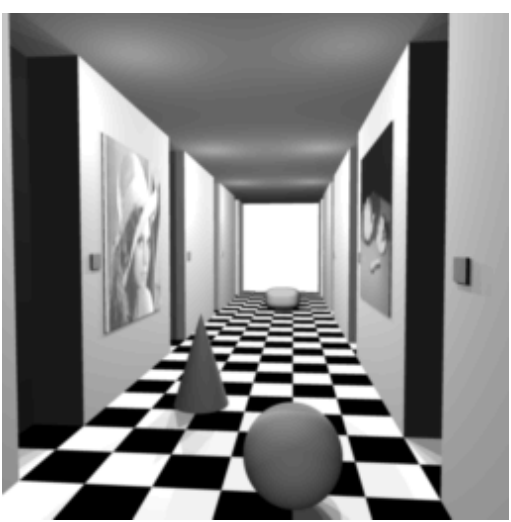

Original left $\bar{x}_{1}$

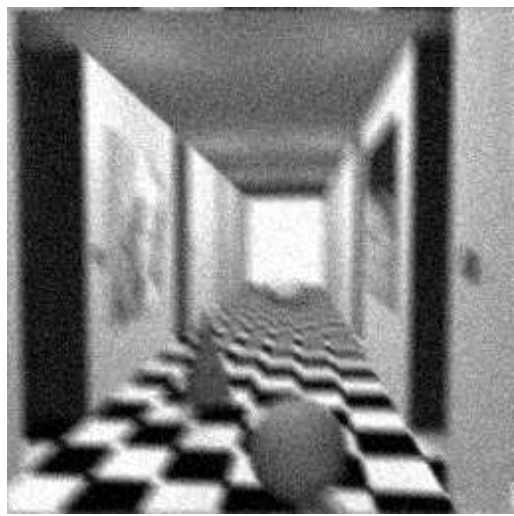

Degraded left $z_{1}$

$\mathrm{SNR}=12.9 \mathrm{~dB}-\mathrm{SSIM}=0.39$

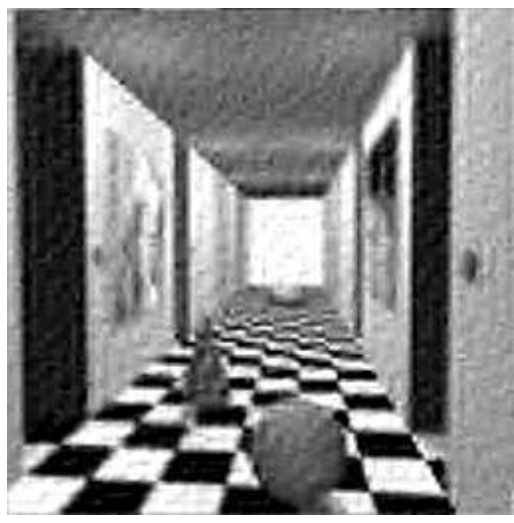

Restored left $x_{1}$ with $\vartheta=0$

$\mathrm{SNR}=15.5 \mathrm{~dB}-\mathrm{SSIM}=0.58$

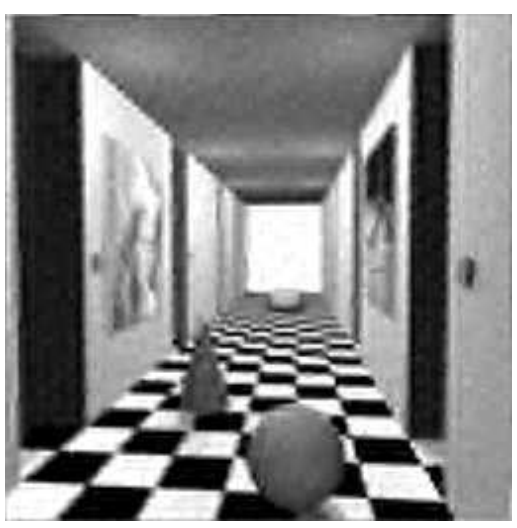

Restored left $x_{1}$ with $\vartheta=1.6 \times 10^{-3}$

$\mathrm{SNR}=17.8 \mathrm{~dB}-\mathrm{SSIM}=0.79$

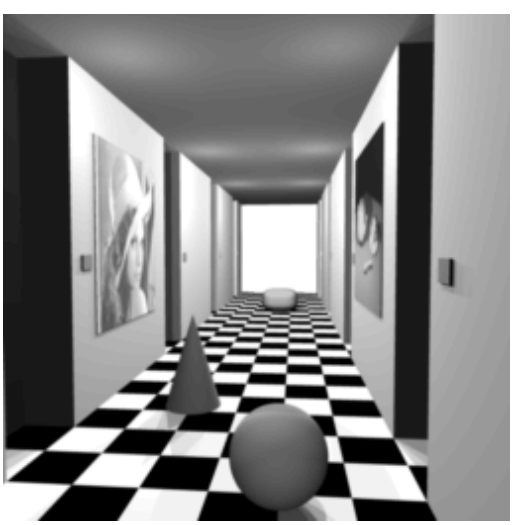

Original right $\bar{x}_{2}$

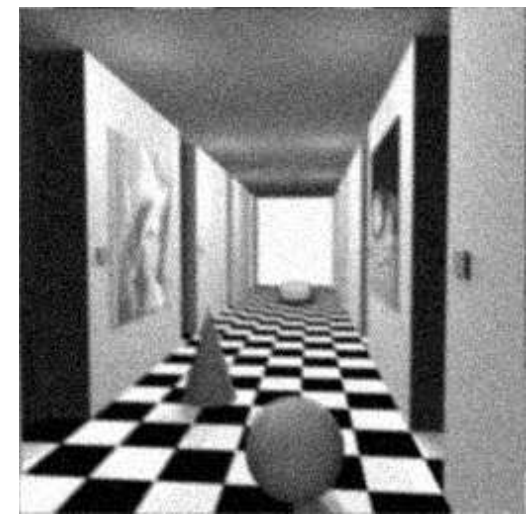

Degraded right $z_{2}$

$\mathrm{SNR}=18.0 \mathrm{~dB}-\mathrm{SSIM}=0.56$

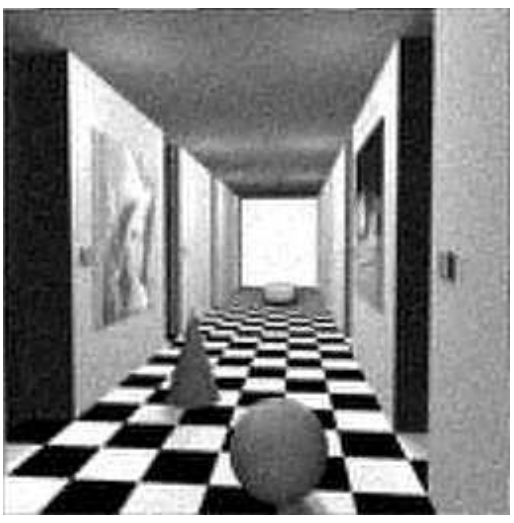

Restored right $x_{2}$ with $\vartheta=0$

$\mathrm{SNR}=19.3 \mathrm{~dB}-\mathrm{SSIM}=0.73$

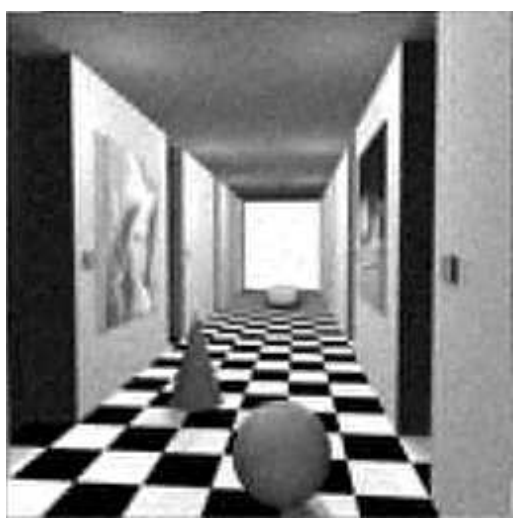

Restored right $x_{2}$ with $\vartheta=1.6 \times 10^{-3}$

$\mathrm{SNR}=19.7 \mathrm{~dB}-\mathrm{SSIM}=0.83$

Figure 1: Stereoscopic image restoration. 
Proof 5.2 The expression (57) follows from straightforward calculus. Now set

$$
\boldsymbol{L}=\left[\begin{array}{cc}
\sigma_{1}^{-1} L_{1} & {[0]} \\
{[0]} & \sigma_{2}^{-1} L_{2}
\end{array}\right] \text { and } \boldsymbol{M}=\sqrt{\vartheta}\left[\begin{array}{cc}
\mathrm{I} & -D \\
{[0]} & {[0]}
\end{array}\right]
$$

Then, using matrix notation, we can write

$$
\nabla f_{2}\left[\begin{array}{l}
x_{1} \\
x_{2}
\end{array}\right]=\left(\boldsymbol{L}^{\top} \boldsymbol{L}+\boldsymbol{M}^{\top} \boldsymbol{M}\right)\left[\begin{array}{l}
x_{1} \\
x_{2}
\end{array}\right]-\boldsymbol{L}^{\top}\left[\begin{array}{l}
\sigma_{1}^{-1} z_{1} \\
\sigma_{2}^{-1} z_{2}
\end{array}\right]
$$

Hence, a Lipschitz constant of $\nabla f_{2}$ is $\left\|\boldsymbol{L}^{\top} \boldsymbol{L}+\boldsymbol{M}^{\top} \boldsymbol{M}\right\|$, where $\|\cdot\|$ denotes the spectral norm. To obtain a tractable bound, we observe that

$$
\begin{aligned}
\left\|\boldsymbol{L}^{\top} \boldsymbol{L}+\boldsymbol{M}^{\top} \boldsymbol{M}\right\| & \leq\left\|\boldsymbol{L}^{\top} \boldsymbol{L}\right\|+\left\|\boldsymbol{M}^{\top} \boldsymbol{M}\right\| \\
& =\|\boldsymbol{L}\|^{2}+\|\boldsymbol{M}\|^{2} \\
& =\|\boldsymbol{L}\|^{2}+\vartheta\left(1+\|D\|^{2}\right) .
\end{aligned}
$$

Now set $\boldsymbol{x}=\left[\begin{array}{ll}x_{1} & x_{2}\end{array}\right]^{\top}$. Then

$$
\begin{aligned}
\|\boldsymbol{L} \boldsymbol{x}\|^{2} & =\sigma_{1}^{-2}\left\|L_{1} x_{1}\right\|^{2}+\sigma_{2}^{-2}\left\|L_{2} x_{2}\right\|^{2} \\
& \leq \sigma_{1}^{-2}\left\|L_{1}\right\|^{2}\left\|x_{1}\right\|^{2}+\sigma_{2}^{-2}\left\|L_{2}\right\|^{2}\left\|x_{2}\right\|^{2} \\
& \leq \max \left\{\sigma_{1}^{-2}\left\|L_{1}\right\|^{2}, \sigma_{2}^{-2}\left\|L_{2}\right\|^{2}\right\}\|\boldsymbol{x}\|^{2} .
\end{aligned}
$$

Hence, $\|\boldsymbol{L}\|^{2} \leq \max \left\{\sigma_{1}^{-2}\left\|L_{1}\right\|^{2}, \sigma_{2}^{-2}\left\|L_{2}\right\|^{2}\right\}$ and (61) yields $\left\|\boldsymbol{L}^{\top} \boldsymbol{L}+\boldsymbol{M}^{\top} \boldsymbol{M}\right\| \leq \beta$.

In view of Proposition 5.1, Problem 5.1 can be solved by the forward-backward algorithm (see Theorem 4.1).

\subsubsection{Numerical experiments}

Experimental results are displayed in Figure 1 for stereoscopic images of size $256 \times 256\left(N=256^{2}\right)$. In this example, $L_{1}$ and $L_{2}$ are periodic convolution operators with motion kernel blur of sizes $7 \times 7$ and $3 \times 3$, respectively. This kind of blur was considered in a related context in [36]. A white Gaussian noise is added corresponding to a blurred signal-to-noise-ratio (BSNR) of $21.6 \mathrm{~dB}$ for $z_{1}$ and $21.8 \mathrm{~dB}$ for $z_{2}$. The observed image $z_{i}$ with $i \in\{1,2\}$ being degraded by an additive noise with variance $\sigma_{i}^{2}$, the BSNR is defined as $10 \log _{10}\left(\left\|L_{i} \bar{x}_{i}\right\|^{2} /\left(N \sigma_{i}^{2}\right)\right)$. In addition, $\left(e_{1, k}\right)_{1 \leq k \leq N}$ and $\left(e_{2, k}\right)_{1 \leq k \leq N}$ are symmlet wavelet orthonormal bases (length 6$)$ over 2 resolution levels. For every $k \in\{1, \ldots, N\}, \phi_{1, k}=\mu_{1, k}|\cdot|^{p_{1, k}}$ and $\phi_{2, k}=\left.\mu_{2, k}|\cdot|\right|^{p_{2, k}}$, where $\left.\left\{\mu_{1, k}, \mu_{2, k}\right\} \subset\right] 0,+\infty[$ and $\left\{p_{1, k}, p_{2, k}\right\} \subset[1,+\infty[$.

The operators $\left(\operatorname{prox}_{\phi_{1, k}}\right)_{1 \leq k \leq N}$ and $\left(\operatorname{prox}_{\phi_{2, k}}\right)_{1 \leq k \leq N}$ can be calculated explicitly [14, Examples 4.2 and 4.4]. The proximity operator of $f_{1}$ can thus be deduced from Proposition 3.3, the separability of this function, and [21, Lemma 2.8 and 2.9]. For every $k \in\{1, \ldots, N\}$, the values of $\mu_{1, k}, \mu_{2, k}, p_{1, k}$, and $p_{2, k}$ are chosen using a maximum likelihood approach in a subband-adaptive 
manner with $p_{1, k}$ and $p_{2, k}$ in $\{1,4 / 3,3 / 2,2\}$. The value of $\vartheta$ is selected so as to maximize the signal-to-noise-ratio (SNR). The SNR between an image $y$ and the original image $\bar{y}$ is defined as $20 \log _{10}(\|\bar{y}\| /\|y-\bar{y}\|)$. In our experiments we also propose to compare the restored images in terms of structural similarity (SSIM) [44]. The SSIM takes on values from -1 to 1 . The value 1 is achieved for two identical images. The disparity map has been estimated by using the method described in [32]. Note that the existence of a solution to Problem 5.1 is secured by the fact that $f_{1}+f_{2}$ is a coercive function in $\Gamma_{0}\left(\mathbb{R}^{N} \oplus \mathbb{R}^{N}\right)$ [21, Propositions 3.1(i) and 5.15(i)]. Thus, Problem 5.1 is a special case of Problem 4.1. In this context, setting $\lambda_{n} \equiv 1$, the forward-backward algorithm assumes the following form.

$$
\begin{aligned}
& \text { Initialization } \\
& \qquad \begin{array}{l}
\sigma_{1}=\sigma_{2}=12 \\
\vartheta=0 \quad \text { or } \quad \vartheta=1.6 \times 10^{-3} \\
\gamma=1.9 /\left(\max \left\{\sigma_{1}^{-2}\left\|L_{1}\right\|^{2}, \sigma_{2}^{-2}\left\|L_{2}\right\|^{2}\right\}+\vartheta\left(1+\|D\|^{2}\right)\right) \\
x_{1,0}=z_{1} \\
x_{2,0}=z_{2}
\end{array} \\
& \text { For } n=0,1, \ldots \\
& \mid \begin{array}{l}
y_{1, n}=\sigma_{1}^{-2} L_{1}^{\top}\left(L_{1} x_{1, n}-z_{1}\right)+\vartheta\left(x_{1, n}-D x_{2, n}\right) \\
y_{2, n}=\sigma_{2}^{-2} L_{2}^{\top}\left(L_{2} x_{2, n}-z_{2}\right)-\vartheta D^{\top}\left(x_{1, n}-D x_{2, n}\right) \\
x_{1, n+1}=\sum_{k=1}^{N}\left(\operatorname{prox}_{\gamma \phi_{1, k}}\left\langle x_{1, n}-\gamma y_{1, n} \mid e_{1, k}\right\rangle\right) e_{1, k} \\
x_{2, n+1}=\sum_{k=1}^{N}\left(\operatorname{prox}_{\gamma \phi_{2, k}}\left\langle x_{2, n}-\gamma y_{2, n} \mid e_{2, k}\right\rangle\right) e_{2, k}
\end{array}
\end{aligned}
$$

When $\vartheta=0$, there is no coupling between the left and right views (images in the third row of Figure 1). As can be observed in Figure 1, the coupling term leads to a significant improvement of the restoration, especially for the most degraded image (bottom-left image).

Using $\vartheta=1.6 \times 10^{-3}$, we compare the forward-backward algorithm of Theorem 4.1 (implemented with $\lambda_{n} \equiv 1$ and $\left.\gamma_{n} \equiv 1.99 / \beta\right)$ to a multicomponent version of the Beck-Teboulle algorithm [9] and a multicomponent version of the Nesterov algorithm [35]. Although, contrary to the forwardbackward algorithm, the Beck-Teboulle and Nesterov algorithms do not insure convergence of the iterates, they are known to provide a theoretically optimal convergence rate for the objective function. However, in this example, their performance appear to be quite comparable on that score (see Figure 2). 


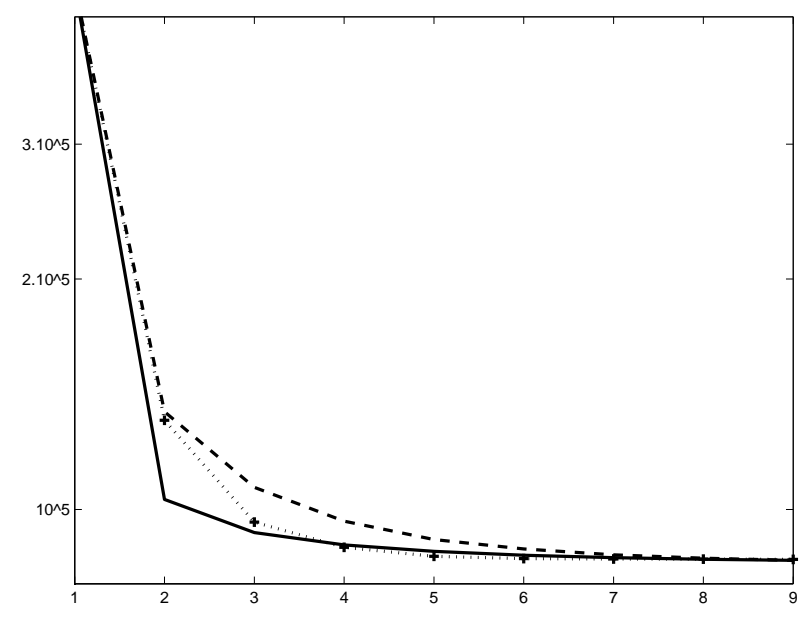

Figure 2: Convergence of the objective function in Problem 5.1 for the forward-backward algorithm (solid line), the Nesterov algorithm (dotted line), and the Beck-Teboulle algorithm (dashed line) versus iteration number.

\subsection{Multispectral image denoising}

\subsubsection{Problem formulation}

A common multispectral image processing problem is to denoise $m$ images $\left(\bar{y}_{i}\right)_{1 \leq i \leq m}$ in $\mathbb{R}^{N}$ from noisy observations $\left(z_{i}\right)_{1 \leq i \leq m}$ given by

$$
(\forall i \in\{1, \ldots, m\}) \quad z_{i}=\bar{y}_{i}+w_{i}
$$

where $\left(w_{i}\right)_{1 \leq i \leq m}$ are realizations of mutually independent zero-mean white Gaussian noise processes with respective variances $\left(\sigma_{i}^{2}\right)_{1 \leq i \leq m}$. Early methods for multispectral image recovery are described in [28]. A tutorial on wavelet-based multispectral denoising can be found in [13].

To solve this denoising problem, we assume that, for every $i \in\{1, \ldots, m\}, \bar{y}_{i}$ satisfies some constraint represented by a nonempty closed convex set $C_{i} \subset \mathbb{R}^{N}$, and that it admits a sparse decomposition in an orthonormal basis $\left(e_{i, k}\right)_{1 \leq k \leq N}$ of $\mathbb{R}^{N}$. In addition, similarities between the images are promoted by penalizing a distance between their components in some orthonormal basis $\left(b_{k}\right)_{1 \leq k \leq N}$ of $\mathbb{R}^{N}$. These considerations lead to the variational problem

$$
\underset{y_{1} \in C_{1}, \ldots, y_{m} \in C_{m}}{\operatorname{minimize}} \sum_{i=1}^{m} \frac{1}{2 \sigma_{i}^{2}}\left\|y_{i}-z_{i}\right\|^{2}+\sum_{i=1}^{m} \sum_{k=1}^{N} \widetilde{\mu}_{i, k}\left|\left\langle y_{i} \mid e_{i, k}\right\rangle\right|+\sum_{i=1}^{m-1} \sum_{j=i+1}^{m} \widetilde{\vartheta}_{i, j} \sum_{k=1}^{N}\left|\left\langle y_{i}-y_{j} \mid b_{k}\right\rangle\right|
$$

where, for every $\left.i \in\{1, \ldots, m\},\left\{\widetilde{\mu}_{i, k}\right\}_{1 \leq k \leq N} \subset\right] 0,+\infty\left[\right.$ and $\left.\left\{\widetilde{\vartheta}_{i, j}\right\}_{i+1 \leq j \leq m} \subset\right] 0,+\infty[$. After appropriate rescaling of the variables, this problem can be reformulated as follows.

Problem 5.2 For every $i \in\{1, \ldots, m\}$, let $\left.\left\{\mu_{i, k}\right\}_{1 \leq k \leq N} \subset\right] 0,+\infty\left[\right.$ and $\left.\left\{\vartheta_{i, j}\right\}_{i+1 \leq j \leq m} \subset\right] 0,+\infty[$. 
The objective is to

$$
\begin{aligned}
\underset{x_{1} \in \mathbb{R}^{N}, \ldots, x_{m} \in \mathbb{R}^{N}}{\operatorname{minimize}} & \frac{p-1}{2} \sum_{i=1}^{m}\left\|x_{i}-\sigma_{i}^{-1} z_{i}\right\|^{2} \\
& +\sum_{i=1}^{m} \sum_{k=1}^{N} \mu_{i, k} \sigma_{i}\left|\left\langle x_{i} \mid e_{i, k}\right\rangle\right| \\
& +\sum_{i=1}^{m-1} \sum_{j=i+1}^{m} \vartheta_{i, j} \sum_{k=1}^{N}\left|\left\langle\sigma_{i} x_{i}-\sigma_{j} x_{j} \mid b_{k}\right\rangle\right| \\
& +\sum_{i=1}^{m} \iota^{\iota} C_{i}\left(\sigma_{i} x_{i}\right) .
\end{aligned}
$$

To cast this problem in the format of Problem 1.1, let us define

$$
J=\left\{(i, j) \in \mathbb{N}^{2} \mid 1 \leq i \leq m-1, i+1 \leq j \leq m\right\}
$$

and

$$
\text { i: } \begin{aligned}
J & \rightarrow\{1, \ldots, m(m-1) / 2\} \\
(i, j) & \mapsto m(i-1)-i(i+1) / 2+j .
\end{aligned}
$$

Moreover, let us set $p=m(m-1) / 2+3$ and

$$
\left\{\begin{aligned}
(\forall(i, j) \in J) \quad f_{\mathrm{i}(i, j)}: & \left(x_{1}, \ldots, x_{m}\right) \mapsto \\
& \vartheta_{i, j} \sum_{k=1}^{N}\left|\left\langle\sigma_{i} x_{i}-\sigma_{j} x_{j} \mid b_{k}\right\rangle\right| \\
f_{p-2}:\left(x_{1}, \ldots, x_{m}\right) & \mapsto \sum_{i=1}^{m} \sum_{k=1}^{N} \mu_{i, k} \sigma_{i}\left|\left\langle x_{i} \mid e_{i, k}\right\rangle\right| \\
f_{p-1}:\left(x_{1}, \ldots, x_{m}\right) & \mapsto \sum_{i=1}^{m} \iota_{C_{i}}\left(\sigma_{i} x_{i}\right) \\
f_{p}:\left(x_{1}, \ldots, x_{m}\right) & \mapsto \frac{p-1}{2} \sum_{i=1}^{m}\left\|x_{i}-\sigma_{i}^{-1} z_{i}\right\|^{2} .
\end{aligned}\right.
$$

Note that, for every $k \in\{1, \ldots, p-2\}$, $\operatorname{dom} f_{k}=\left(\mathbb{R}^{N}\right)^{m}$ and $\operatorname{dom} f_{p-1}=\sigma_{1}^{-1} C_{1} \times \cdots \times \sigma_{m}^{-1} C_{m}$. Hence, since the sets $\left(C_{i}\right)_{1 \leq i \leq m}$ are nonempty, (49) holds and Problem 5.2 can be solved by the Dykstra-like algorithm presented in Theorem 4.10, with $\mathcal{H}_{1}=\cdots=\mathcal{H}_{m}=\mathbb{R}^{N}$. An explicit form of the proximity operators of the functions $\left(f_{k}\right)_{1 \leq k \leq m(m-1) / 2}$ can be deduced from Proposition 3.9. Indeed, for every $(i, j) \in J$, we can set in this proposition $\mathcal{H}_{1}=\cdots=\mathcal{H}_{m}=\mathcal{G}=\mathbb{R}^{N},(\forall k \in$ $\{1, \ldots, N\})$ and $\phi_{k}=\vartheta_{i, j}|\cdot|$, and define the matrices $\left(L_{\ell}\right)_{1 \leq \ell \leq m}$ in $\mathbb{R}^{N \times N}$ as

$$
(\forall \ell \in\{1, \ldots, m\}) \quad L_{\ell}= \begin{cases}\sigma_{\ell} \mathrm{I}, & \text { if } \ell=i ; \\ -\sigma_{\ell} \mathrm{I}, & \text { if } \ell=j ; \\ 0 & \text { otherwise }\end{cases}
$$


Finally, the proximity operator of $f_{p-2}$ can be derived from Proposition 3.3 combined with the separability of this function, [21, Lemma 2.8 and 2.9], and [14, Example 4.2]. The proximity operator of $f_{p-1}$ is provided in Example 3.1.

\subsubsection{Numerical experiments}

Figure 3 shows the results obtained on a multispectral image of size $256 \times 256\left(N=256^{2}\right)$ with 3 channels $(m=3)$ and pixel values in the range $[0,255]$. These images are corrupted by white Gaussian noises with standard deviations $\sigma_{1}=11, \sigma_{2}=12$, and $\sigma_{3}=13$ (the corresponding SNR values are indicated in Figure 3). On the other hand, $\left(b_{k}\right)_{1 \leq k \leq N}$ is the Haar orthonormal wavelet basis on 3 resolution levels and, for every $i \in\{1, \ldots, m\},\left(e_{i, k}\right)_{1 \leq k \leq N}$ are symmlet orthonormal wavelet bases (length 6 ) on 3 resolution levels. The values of the regularization parameters $\left(\left(\mu_{i, k}\right)_{1 \leq i \leq 3}\right)_{1 \leq k \leq N}$ (chosen subband-adaptive by a maximum likelihood approach), and of the coupling parameters $\vartheta_{1,2}$, $\vartheta_{1,3}$, and $\vartheta_{2,3}$ are selected so as to maximize the SNR. For every $i \in\{1, \ldots, m\}, C_{i}=[0,255]^{N}$ models the constraint on the range of pixel values. The resulting Dykstra-like algorithm is described below. 


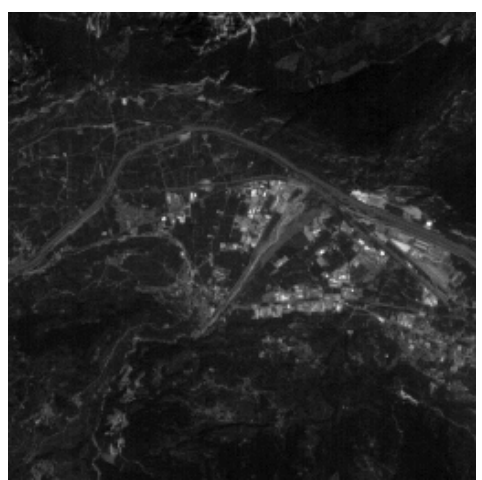

Original image $\bar{y}_{1}$

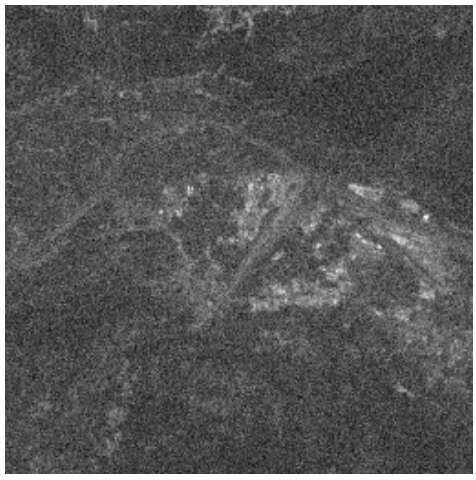

Degraded image $z_{1}$

$\mathrm{SNR}=16.2 \mathrm{~dB}-\mathrm{SSIM}=0.47$

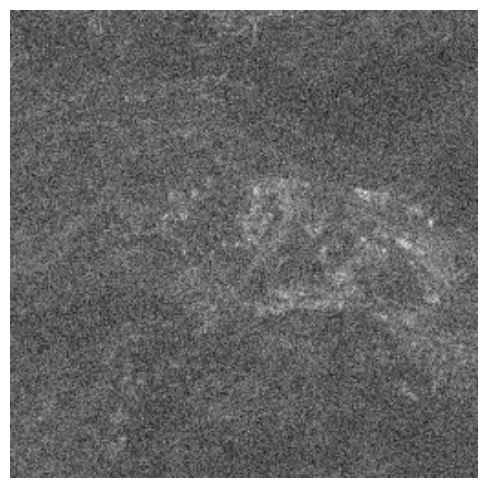

Degraded image $z_{2}$

$\mathrm{SNR}=8.28 \mathrm{~dB}-\mathrm{SSIM}=0.38$

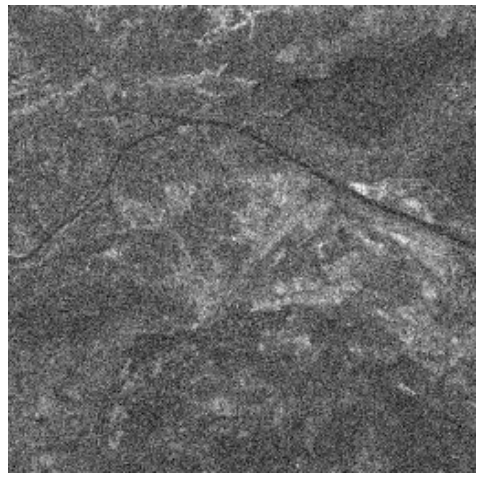

Degraded image $z_{3}$

$\mathrm{SNR}=7.08 \mathrm{~dB}-\mathrm{SSIM}=0.45$

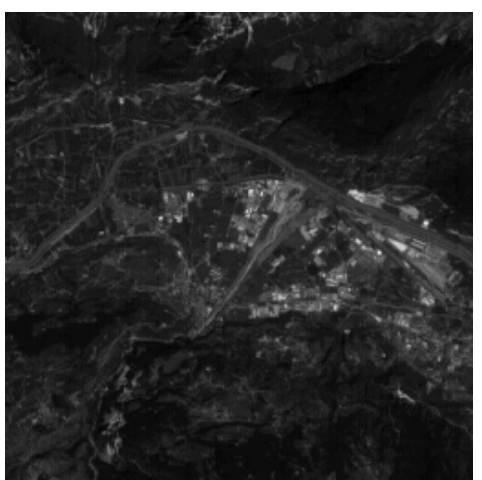

Original image $\bar{y}_{2}$

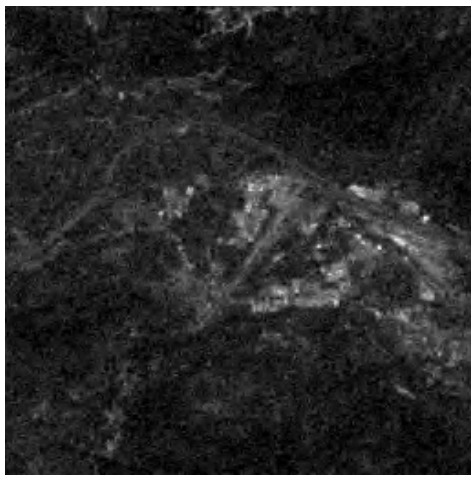

Restored $y_{1}$ without coupling term $\mathrm{SNR}=22.3 \mathrm{~dB}-\mathrm{SSIM}=0.78$

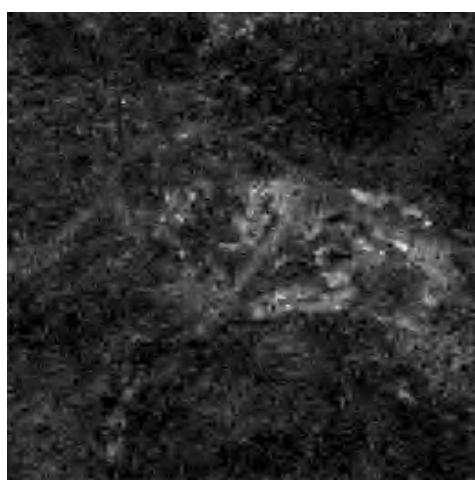

Restored $y_{2}$ without coupling term $\mathrm{SNR}=17.4 \mathrm{~dB}-\mathrm{SSIM}=0.85$

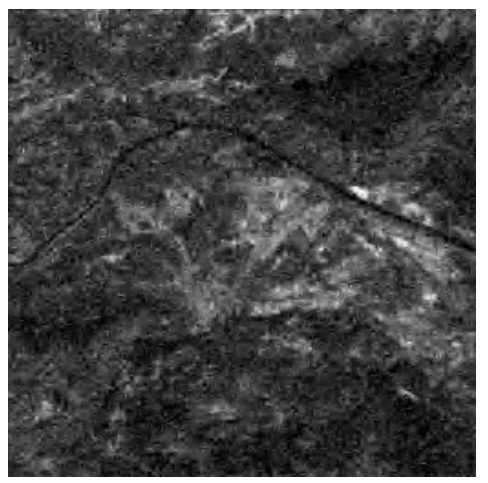

Restored $y_{3}$ without coupling term $\mathrm{SNR}=13.22^{2} \mathrm{~dB}-\mathrm{SSIM}=0.75$

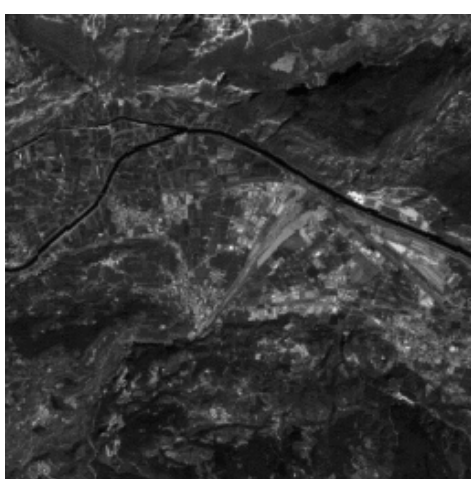

Original image $\bar{y}_{3}$

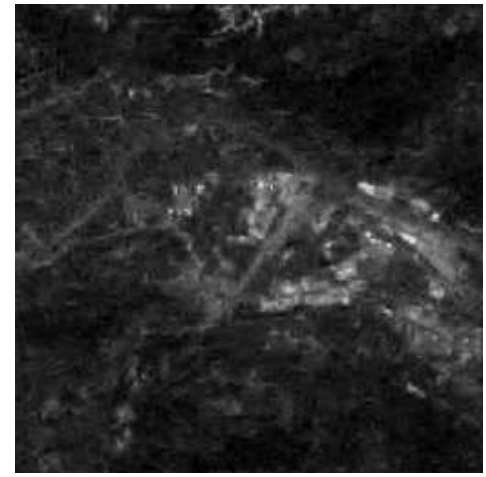

Restored $y_{1}$ with coupling term $\mathrm{SNR}=24.2 \mathrm{~dB}-\mathrm{SSIM}=0.87$

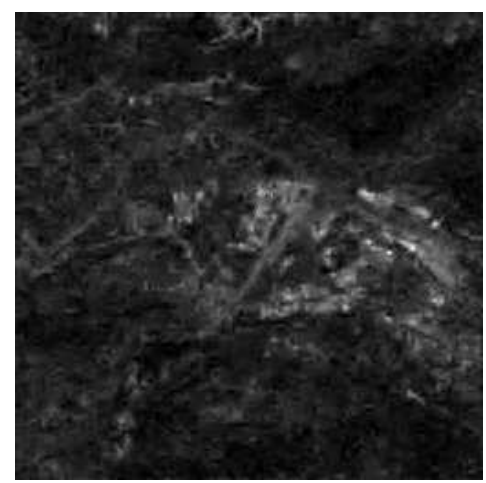

Restored $y_{2}$ with coupling term $\mathrm{SNR}=19.3 \mathrm{~dB}-\mathrm{SSIM}=0.91$

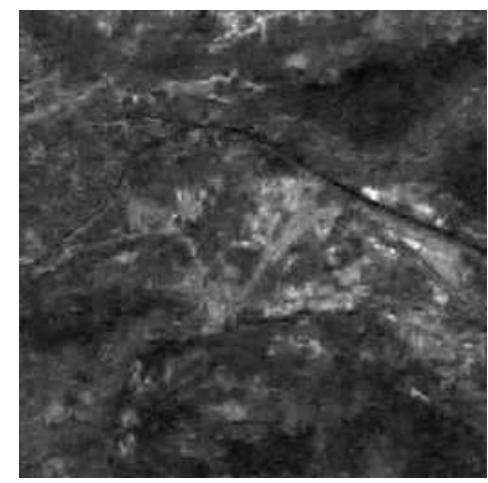

Restored $y_{3}$ with coupling term $\mathrm{SNR}=14.7 \mathrm{~dB}-\mathrm{SSIM}=0.82$

Figure 3: Multispectral restoration. 


\section{Initialization}

$$
\mid \begin{aligned}
& \sigma_{1}=11 ; \sigma_{2}=12 ; \sigma_{3}=13 \\
& y_{1,1,0}=\ldots=y_{1,5,0}=x_{1,0}=z_{1} \\
& y_{2,1,0}=\ldots=y_{2,5,0}=x_{2,0}=z_{2} \\
& y_{3,1,0}=\ldots=y_{3,5,0}=x_{3,0}=z_{3} \\
& \alpha_{1,2}=\sigma_{1}^{2}+\sigma_{2}^{2} \\
& \alpha_{1,3}=\sigma_{1}^{2}+\sigma_{3}^{2} \\
& \alpha_{2,3}=\sigma_{2}^{2}+\sigma_{3}^{2}
\end{aligned}
$$

For $n=0,1, \ldots$

$$
\begin{aligned}
& u_{1,1, n}=y_{1,1, n}+\alpha_{1,2}^{-1} \sigma_{1} \sum_{k=1}^{N}\left(\operatorname{prox}_{\alpha_{1,2} \vartheta_{1,2}|\cdot|}\left\langle\sigma_{1} y_{1,1, n}-\sigma_{2} y_{2,1, n} \mid b_{k}\right\rangle+\left\langle\sigma_{1} y_{1,1, n}-\sigma_{2} y_{2,1, n} \mid b_{k}\right\rangle\right) b_{k} \\
& u_{2,1, n}=y_{2,1, n}-\alpha_{1,2}^{-1} \sigma_{2} \sum_{k=1}^{N}\left(\operatorname{prox}_{\alpha_{1,2} \vartheta_{1,2}|\cdot|}\left\langle\sigma_{1} y_{1,1, n}-\sigma_{2} y_{2,1, n} \mid b_{k}\right\rangle+\left\langle\sigma_{1} y_{1,1, n}-\sigma_{2} y_{2,1, n} \mid b_{k}\right\rangle\right) b_{k} \\
& u_{3,1, n}=y_{3,1, n} \\
& u_{1,2, n}=y_{1,2, n}+\alpha_{1,3}^{-1} \sigma_{1} \sum_{k=1}^{N}\left(\operatorname{prox}_{\alpha_{1,3} \vartheta_{1,3}|\cdot|}\left\langle\sigma_{1} y_{1,2, n}-\sigma_{3} y_{3,2, n} \mid b_{k}\right\rangle+\left\langle\sigma_{1} y_{1,2, n}-\sigma_{3} y_{3,2, n} \mid b_{k}\right\rangle\right) b_{k} \\
& u_{2,2, n}=y_{2,2, n} \\
& u_{3,2, n}=y_{3,2, n}-\alpha_{1,3}^{-1} \sigma_{3} \sum_{k=1}^{N}\left(\operatorname{prox}_{\alpha_{1,3} \vartheta_{1,3}|\cdot|}\left\langle\sigma_{1} y_{1,2, n}-\sigma_{3} y_{3,2, n} \mid b_{k}\right\rangle+\left\langle\sigma_{1} y_{1,2, n}-\sigma_{3} y_{3,2, n} \mid b_{k}\right\rangle\right) b_{k} \\
& u_{1,3, n}=y_{1,3, n} \\
& u_{2,3, n}=y_{2,3, n}+\alpha_{2,3}^{-1} \sigma_{2} \sum_{k=1}^{N}\left(\operatorname{prox}_{\alpha_{2,3} \vartheta_{2,3}|\cdot|}\left\langle\sigma_{2} y_{2,3, n}-\sigma_{3} y_{3,3, n} \mid b_{k}\right\rangle+\left\langle\sigma_{2} y_{2,3, n}-\sigma_{3} y_{3,3, n} \mid b_{k}\right\rangle\right) b_{k} \\
& u_{3,3, n}=y_{3,3, n}-\alpha_{2,3}^{-1} \sigma_{3} \sum_{k=1}^{N}\left(\operatorname{prox}_{\alpha_{2,3} \vartheta_{2,3}|\cdot|}\left\langle\sigma_{2} y_{2,3, n}-\sigma_{3,3} y_{3,3, n} \mid b_{k}\right\rangle+\left\langle\sigma_{2} y_{2,3, n}-\sigma_{3} y_{3,3, n} \mid b_{k}\right\rangle\right) b_{k} \\
& u_{1,4, n}=\sum_{k=1}^{N}\left(\operatorname{prox}_{\mu_{1, k} \sigma_{1}|\cdot|}\left\langle y_{1,4, n} \mid e_{1, k}\right\rangle\right) e_{1, k} \\
& u_{2,4, n}=\sum_{k=1}^{N}\left(\operatorname{prox}_{\mu_{2, k} \sigma_{2}|\cdot|}\left\langle y_{2,4, n} \mid e_{2, k}\right\rangle\right) e_{2, k} \\
& u_{3,4, n}=\sum_{k=1}^{N}\left(\operatorname{prox}_{\mu_{3, k} \sigma_{3}|\cdot|}\left\langle y_{3,4, n} \mid e_{3, k}\right\rangle\right) e_{3, k} \\
& u_{1,5, n}=P_{C_{1}}\left(\sigma_{1} y_{1,5, n}\right) \\
& u_{2,5, n}=P_{C_{2}}\left(\sigma_{2} y_{2,5, n}\right) \\
& u_{3,5, n}=P_{C_{3}}\left(\sigma_{3} y_{3,5, n}\right) \\
& x_{1, n+1}=\left(u_{1,1, n}+u_{1,2, n}+u_{1,3, n}+u_{1,4, n}+u_{1,5, n}\right) / 5 \\
& x_{2, n+1}=\left(u_{2,1, n}+u_{2,2, n}+u_{2,3, n}+u_{2,4, n}+u_{2,5, n}\right) / 5 \\
& x_{3, n+1}=\left(u_{3,1, n}+u_{3,2, n}+u_{3,3, n}+u_{3,4, n}+u_{3,5, n}\right) / 5 \\
& F_{j}=1, \ldots, 5 \\
& \begin{array}{l}
y_{1, j, n+1}=x_{1, n+1}+y_{1, j, n}-u_{1, j, n} \\
y_{2, j, n+1}=x_{2, n+1}+y_{2, j, n}-u_{2, j, n} \\
y_{3, j, n+1}=x_{3, n+1}+y_{3, j, n}-u_{3, j, n}
\end{array}
\end{aligned}
$$


It can be observed from the images displayed on the second and third columns of Figure 3 that the introduction of the coupling term has a significant influence on denoising performance. Moreover, in our experiments, we observed that better results were obtained when different bases $\left(b_{k}\right)_{1 \leq k \leq N},\left(e_{1, k}\right)_{1 \leq k \leq N}, \ldots,\left(e_{m, k}\right)_{1 \leq k \leq N}$ were employed.

It turns out that, in this particular problem, an alternative solution method is PPXA (see Theorem 4.7) applied to the minimization of the sum of the $m(m-1) / 2+2$ functions $f_{1}, f_{2}$, $\ldots, f_{p-2}$, and $f_{p-1}+f_{p}$ defined in (68). The proximity operator of the latter is given by [21, Lemma 2.6(i)]. Indeed, the qualification condition (see (48)) is satisfied since $\operatorname{dom} f_{1}=\cdots=$ $\operatorname{dom} f_{p-2}=\left(\mathbb{R}^{N}\right)^{m}$ and, $\left.(\forall i \in\{1, \ldots, m\}) \operatorname{int} C_{i}=\right] 0,255[\neq \varnothing$. The choice of the PPXA parameters has been optimized empirically for speed of convergence and set to $\lambda_{n} \equiv 1.3, \gamma=1$, and $\omega_{1}=\cdots=\omega_{p-1}=1 /(p-1)$. In Figure $4,\left\|\boldsymbol{x}_{n}-\boldsymbol{x}_{\infty}\right\| /\left\|\boldsymbol{x}_{0}-\boldsymbol{x}_{\infty}\right\|$ is plotted as a function of computation time, where $\left(\boldsymbol{x}_{n}\right)_{n \in \mathbb{N}}=\left(\left(x_{1, n}, x_{2, n}, x_{3, n}\right)\right)_{n \in \mathbb{N}}$ is the sequence generated by an algorithm and $\boldsymbol{x}_{\infty}$ is the unique solution to Problem 5.2. In our experiments, 500 iterations were used to produce this solution.

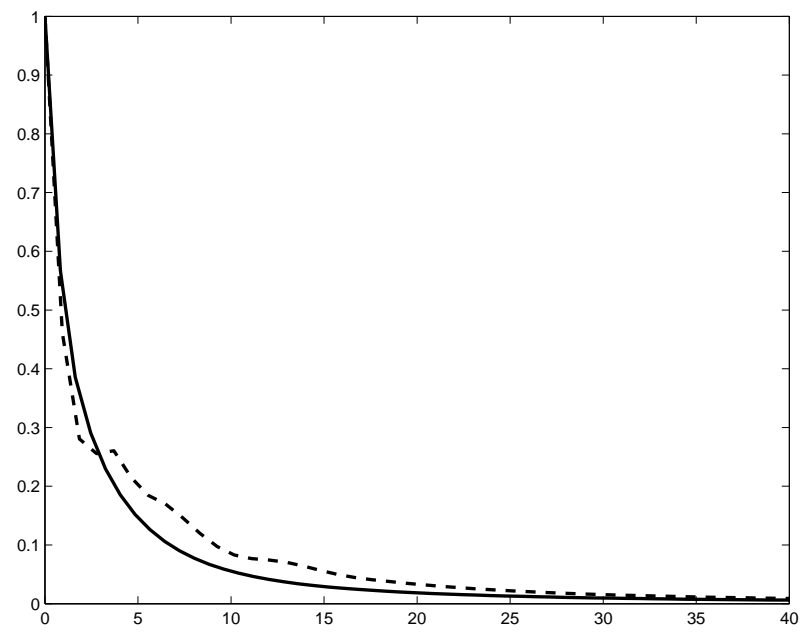

Figure 4: Problem 5.2: Convergence profiles of the Dykstra-like algorithm (solid line) and of PPXA (dashed line) versus computation time in seconds.

\subsection{Structure-texture image decomposition}

An important problem in image processing is to decompose an image into elementary structures. In the context of denoising, this decomposition was investigated in [38] with a total variation potential. In [31], a different potential was used to better penalize strongly oscillating components. The resulting variational problem is not straightforward. Numerical methods were proposed in [4, 41] and experiments were performed for image denoising and analysis problems based on a geometrytexture decomposition. Another challenging problem is to extract meaningful components from a blurred and noise-corrupted image. In the presence of additive Gaussian noise, a decomposition into geometry and texture components is proposed in $[2,23]$. The method developed in the present paper, will make it possible to consider general (not necessarily additive and Gaussian) noise models 
and arbitrary linear degradation operators. We consider a simple geometry-texture decomposition from a degraded observation.

\subsubsection{Problem formulation}

In this experiment, the observed image $z \in \mathbb{R}^{N}$ is obtained by multiplying the original image $\bar{x} \in \mathbb{R}^{N}$ with a matrix $T \in \mathbb{R}^{N \times N}$, which models a blur, and corrupting $T \bar{x}$ by a Poisson noise with scaling parameter $\alpha$. It is assumed that

$T$ has its entries in $[0,+\infty[$ andeach of its rows is nonzero.

The inverse problem we address is to obtain the decomposition of $\bar{x}$ into the sum of a geometry and a texture component, say

$$
\bar{x}=R_{1}\left(\bar{x}_{1}\right)+R_{2}\left(\bar{x}_{2}\right),
$$

where $R_{1}: \mathbb{R}^{N_{1}} \mapsto \mathbb{R}^{N}$ and $R_{2}: \mathbb{R}^{N_{2}} \mapsto \mathbb{R}^{N}$ are known operators. The vectors $\bar{x}_{1} \in \mathbb{R}^{N_{1}}$ and $\bar{x}_{2} \in \mathbb{R}^{N_{2}}$ to be estimated parameterize, respectively, the geometry and the texture components.

We consider a simple instance of (71) involving a linear mixture: $N_{1}=N, R_{1}: x_{1} \mapsto x_{1}$, and $R_{2}: x_{2} \mapsto F^{\top} x_{2}$, where $F^{\top} \in \mathbb{R}^{N \times K}$ is a linear tight frame synthesis operator. In other words, the information regarding the texture component pertains to the coefficients $\bar{x}_{2}$ of its decomposition in the frame. The tightness condition implies that

$$
\left.F^{\top} F=\nu \mathrm{Id} \text {, for some } \nu \in\right] 0,+\infty[.
$$

Thus, the original image is decomposed as $\bar{x}=\bar{x}_{1}+F^{\top} \bar{x}_{2}$. It is known a priori that $\bar{x} \in C_{1} \cap C_{2}$, where

$$
C_{1}=[0,255]^{N}
$$

models the constraint on the range of pixel values, and

$$
C_{2}=\left\{\left.x \in \mathbb{R}^{N}\left|\widehat{x}=\left(\eta_{k}\right)_{1 \leq k \leq N}, \sum_{k \in \mathbb{I}}\right| \eta_{k}\right|^{2} \leq \delta\right\},
$$

for some $\delta \in] 0,+\infty[$, models an energy constraint on the 2-D DFT $\widehat{\bar{x}}$ of the original image in some low frequency band $\mathbb{I} \subset\{1, \ldots, N\}$. In addition, to limit the total variation [11] of the geometrical component, the potential $x \mapsto \psi(H x, V x)$ is used, with

$$
\psi:\left(\left(\eta_{k}\right)_{1 \leq k \leq N},\left(\zeta_{k}\right)_{1 \leq k \leq N}\right) \mapsto \chi \sum_{k=1}^{N} \sqrt{\left|\eta_{k}\right|^{2}+\left|\zeta_{k}\right|^{2}},
$$

where $H \in \mathbb{R}^{N \times N}$ and $V \in \mathbb{R}^{N \times N}$ are matrix representations of the horizontal and vertical discrete differentiation operators, respectively, and where $\chi \in] 0,+\infty[$. Furthermore, to promote sparsity in the frame of the texture component of the image, the potential

$$
h:\left(\eta_{k}\right)_{1 \leq k \leq K} \mapsto \sum_{k=1}^{K} \tau_{k}\left|\eta_{k}\right|
$$


is introduced, where $\left.\left\{\tau_{k}\right\}_{1 \leq k \leq K} \subset\right] 0,+\infty$ [. Finally, as a data fidelity term well adapted to Poisson noise, we employ the generalized Kullback-Leibler divergence with a scaling parameter $\alpha \in] 0,+\infty[$. Upon setting $z=\left(\zeta_{k}\right)_{1 \leq k \leq N}$, this leads to the function

$$
g:\left(\xi_{k}\right)_{1 \leq k \leq N} \mapsto \sum_{k=1}^{N} \phi_{k}\left(\xi_{k}\right)
$$

where, for every $k \in\{1, \ldots, K\}$,

$$
\begin{aligned}
\left.\left.\phi_{k}: \mathbb{R} \rightarrow\right]-\infty,+\infty\right] & \\
\xi & \mapsto \begin{cases}-\zeta_{k} \ln (\xi)+\alpha \xi, & \text { if } \zeta_{k} \geq 0 \text { and } \xi>0 ; \\
0, & \text { if } \xi=0 ; \\
+\infty, & \text { otherwise. }\end{cases}
\end{aligned}
$$

Altogether, the variational problem is to

$$
\underset{\substack{x_{1} \in \mathbb{R}^{N}, x_{2} \in \mathbb{R}^{K} \\ x_{1}+F^{\top} x_{2} \in C_{1} \\ x_{1}+F^{\top} x_{2} \in C_{2}}}{\operatorname{minimize}} \psi\left(H x_{1}, V x_{1}\right)+h\left(x_{2}\right)+g\left(T x_{1}+T F^{\top} x_{2}\right) .
$$

This problem is a particular case of (2) with $m=2, p=4$, and

$$
\left\{\begin{array}{l}
f_{1}:\left(x_{1}, x_{2}\right) \mapsto \psi\left(H x_{1}, V x_{1}\right)+h\left(x_{2}\right), \\
f_{2}:\left(x_{1}, x_{2}\right) \mapsto g\left(T x_{1}+T F^{\top} x_{2}\right), \\
f_{3}:\left(x_{1}, x_{2}\right) \mapsto \iota_{C_{1}}\left(x_{1}+F^{\top} x_{2}\right), \\
f_{4}:\left(x_{1}, x_{2}\right) \mapsto \iota_{C_{2}}\left(x_{1}+F^{\top} x_{2}\right) .
\end{array}\right.
$$

However, since the operators $\left(\operatorname{prox}_{f_{i}}\right)_{1 \leq i \leq 4}$ are not easily implementable, we cannot apply directly Theorems 4.1, 4.4, or 4.7. To circumvent this difficulty, a strategy is to decompose (79) into an equivalent problem by introducing auxiliary variables.

A first equivalent problem to (79) is

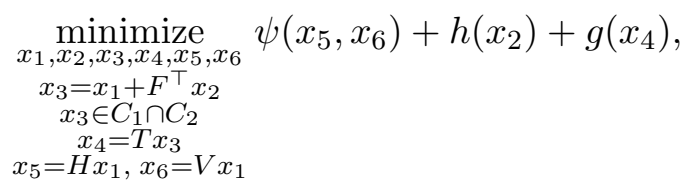

where we have introduced the auxiliary variables $\left(x_{3}, x_{4}, x_{5}, x_{6}\right) \in \mathbb{R}^{N} \oplus \mathbb{R}^{N} \oplus \mathbb{R}^{N} \oplus \mathbb{R}^{N}$. Problem (81) is a particular case of (2) with $m=6, p=3$, and

$$
\left\{\begin{array}{l}
f_{1}:\left(x_{1}, \ldots, x_{6}\right) \mapsto h\left(x_{2}\right)+\iota_{C_{1}}\left(x_{3}\right)+g\left(x_{4}\right)+\psi\left(x_{5}, x_{6}\right), \\
f_{2}:\left(x_{1}, \ldots, x_{6}\right) \mapsto \iota_{C_{2}}\left(x_{3}\right), \\
f_{3}:\left(x_{1}, \ldots, x_{6}\right) \mapsto \iota_{\{0\}}\left(x_{1}+F^{\top} x_{2}-x_{3}\right)+\iota_{\{0\}}\left(T x_{3}-x_{4}\right)+\iota_{\{0\}}\left(H x_{1}-x_{5}\right)+\iota_{\{0\}}\left(V x_{1}-x_{6}\right) .
\end{array}\right.
$$

In this formulation, the rôle of $f_{3}$ is to impose the constraints $x_{1}+F^{\top} x_{2}=x_{3}, T x_{3}=x_{4}, H x_{1}=x_{5}$, and $V x_{1}=x_{6}$. As seen in Example 3.1, $\operatorname{prox}_{\iota_{C_{1}}}=P_{C_{1}}$ and $\operatorname{prox}_{\iota_{C_{2}}}=P_{C_{2}}$. On the other hand, the 
proximity operators of $\psi, h$, and $g$ can be obtained from [19, Proposition 2.8(i)], [21, Example 2.16], and [17, Example 30], respectively. In turn, since $f_{1}$ is separable, its proximity operator follows straightforwardly componentwise. Now set

$$
\boldsymbol{L}_{1}=\left[\begin{array}{cccccc}
\mathrm{I} & F^{\top} & -\mathrm{I} & {[0]} & {[0]} & {[0]} \\
{[0]} & {[0]} & T & -\mathrm{I} & {[0]} & {[0]} \\
H & {[0]} & {[0]} & {[0]} & -\mathrm{I} & {[0]} \\
V & {[0]} & {[0]} & {[0]} & {[0]} & -\mathrm{I}
\end{array}\right]
$$

It follows from (82) and (83) that $f_{3}=\iota_{\text {ker } \boldsymbol{L}_{1}}$, where ker $\boldsymbol{L}_{1}=\left\{\boldsymbol{x} \in \mathcal{H} \mid \boldsymbol{L}_{1} \boldsymbol{x}=\mathbf{0}\right\}$. Hence, by Example 3.1 and [24, Chapter 8],

$$
\operatorname{prox}_{f_{3}}=P_{\text {ker } \boldsymbol{L}_{1}}=\mathrm{I}-\boldsymbol{L}_{1}^{\top}\left(\boldsymbol{L}_{1} \boldsymbol{L}_{1}^{\top}\right)^{-1} \boldsymbol{L}_{1} .
$$

Under the assumption that the matrices $T, H$, and $V$ are block-circulant with circulant blocks, they are diagonalized by the 2-D DFT. Hence, combining (84), (83), and (72) we deduce that prox $_{f_{3}}$ is computable explicitly. On the other hand, it follows from (82), (76), (73), (77), (75), (74), and (83) that

$$
\left\{\begin{array}{l}
\text { ridom } \left.f_{1}=\mathbb{R}^{N} \times \mathbb{R}^{K} \times \operatorname{int} C_{1} \times\right] 0,+\infty\left[^{N} \times \mathbb{R}^{N} \times \mathbb{R}^{N}\right. \\
\text { ridom } f_{2}=\mathbb{R}^{N} \times \mathbb{R}^{K} \times \operatorname{int} C_{2} \times \mathbb{R}^{N} \times \mathbb{R}^{N} \times \mathbb{R}^{N} \\
\text { ridom } f_{3}=\operatorname{ker} \boldsymbol{L}_{1} .
\end{array}\right.
$$

Hence, qualification condition (48) reduces to

$$
\left(\exists\left(x_{1}, \ldots, x_{6}\right) \in \operatorname{ker} \boldsymbol{L}_{1}\right)\left\{\begin{array}{l}
x_{3} \in \operatorname{int} C_{1} \cap \operatorname{int} C_{2} \\
\left.x_{4} \in\right] 0,+\infty\left[^{N}\right.
\end{array}\right.
$$

which is equivalent to

$$
\begin{aligned}
\left(\exists\left(x_{1}, \ldots, x_{6}\right) \in \mathbb{R}^{N} \times \mathbb{R}^{K} \times \mathbb{R}^{N} \times \mathbb{R}^{N} \times \mathbb{R}^{N} \times \mathbb{R}^{N}\right) & \left\{\begin{array}{l}
x_{1}+F^{\top} x_{2}=x_{3} \in \operatorname{int} C_{1} \cap \operatorname{int} C_{2}, \\
\left.T x_{3}=x_{4} \in\right] 0,+\infty\left[^{N}\right. \\
H x_{1}=x_{5} \\
V x_{1}=x_{6} .
\end{array}\right.
\end{aligned}
$$

This condition is satisfied if

$$
\left.T\left(\operatorname{int}\left(C_{1} \cap C_{2}\right)\right) \cap\right] 0,+\infty\left[^{N} \neq \varnothing .\right.
$$

Indeed, let $\left.y \in T\left(\operatorname{int}\left(C_{1} \cap C_{2}\right)\right) \cap\right] 0,+\infty\left[^{N}\right.$. Then there exists $x \in \operatorname{int}\left(C_{1} \cap C_{2}\right)$ such that $T x=y \in$ ] $0,+\infty\left[{ }^{N}\right.$. Hence, for every $x_{2} \in \mathbb{R}^{K}$, if we set $x_{3}=x, x_{4}=y=T x_{3}, x_{1}=x_{3}-F^{\top} x_{2}, x_{5}=H x_{1}$, and $V x_{1}=x_{6},(87)$ is seen to hold. Since (73) and (74) yield $\operatorname{int}\left(C_{1} \cap C_{2}\right) \neq \varnothing$, we deduce from (70) that (88) (and hence (48)) is satisfied. Thus, (81) can be solved by PPXA (see Theorem 4.7 and Remark 4.9).

Another equivalent formulation of (79) is

$$
\begin{gathered}
\underset{x_{1}, x_{2}, x_{3}, x_{4}, x_{5}, x_{6}, x_{7}}{\operatorname{minimize}} \psi\left(x_{5}, x_{6}\right)+h\left(x_{2}\right)+g\left(x_{4}\right), \\
x_{3}=x_{1}+F^{\top} x_{2} \\
x_{4}=T x_{3} \\
x_{5}=H x_{1}, x_{6}=V x_{1} \\
x_{7}=x_{3}=C_{2} \\
x_{3} \in C_{1}, x_{7} \in C_{2}
\end{gathered}
$$


where the additional auxiliary variable $x_{7} \in \mathbb{R}^{N}$ has been introduced. Problem (89) is a particular case of (2) with $m=7, p=2$, and

$$
\left\{\begin{aligned}
f_{1}:\left(x_{1}, \ldots, x_{7}\right) \mapsto & h\left(x_{2}\right)+\iota_{C_{1}}\left(x_{3}\right)+g\left(x_{4}\right)+\psi\left(x_{5}, x_{6}\right)+\iota_{C_{2}}\left(x_{7}\right) \\
f_{2}:\left(x_{1}, \ldots, x_{7}\right) \mapsto & \iota_{\{0\}}\left(x_{1}+F^{\top} x_{2}-x_{3}\right)+\iota_{\{0\}}\left(T x_{3}-x_{4}\right)+\iota_{\{0\}}\left(H x_{1}-x_{5}\right) \\
& +\iota_{\{0\}}\left(V x_{1}-x_{6}\right)+\iota_{\{0\}}\left(x_{3}-x_{7}\right) .
\end{aligned}\right.
$$

As previously observed, since the proximity operators of $\psi, h, g, \iota_{C_{1}}$, and $\iota_{C_{2}}$ are easily computable, so is $\operatorname{prox}_{f_{1}}$. Furthermore, if we set

$$
\boldsymbol{L}_{2}=\left[\begin{array}{ccccccc}
\mathrm{I} & F^{\top} & -\mathrm{I} & {[0]} & {[0]} & {[0]} & {[0]} \\
{[0]} & {[0]} & T & -\mathrm{I} & {[0]} & {[0]} & {[0]} \\
H & {[0]} & {[0]} & {[0]} & -\mathrm{I} & {[0]} & {[0]} \\
V & {[0]} & {[0]} & {[0]} & {[0]} & -\mathrm{I} & {[0]} \\
{[0]} & {[0]} & \mathrm{I} & {[0]} & {[0]} & {[0]} & -\mathrm{I}
\end{array}\right]
$$

it can be deduced from (90) that the proximity operator of $f_{2}=\iota_{\mathrm{ker}} \boldsymbol{L}_{2}$ can be computed like that of $\iota_{\mathrm{ker}} \boldsymbol{L}_{1}$. We derive from (90), (76), (73), (77), (75), (74), and (91) that

$$
\left\{\begin{array}{l}
\left.\operatorname{ridom} f_{1}=\mathbb{R}^{N} \times \mathbb{R}^{K} \times \operatorname{int} C_{1} \times\right] 0,+\infty\left[{ }^{N} \times \mathbb{R}^{N} \times \mathbb{R}^{N} \times \operatorname{int} C_{2}\right. \\
\operatorname{ridom} f_{2}=\operatorname{ker} \boldsymbol{L}_{2} .
\end{array}\right.
$$

Hence, arguing as above, (45) reduces to (88), which is seen to be satisfied. This shows that (89) can be solved by the Douglas-Rachford algorithm (see Theorem 4.4 and Remark 4.6(ii)).

\subsubsection{Numerical experiments}

Figure 5 shows the results of the decomposition into geometry and texture components of an electron microscopy image of size $512 \times 512\left(N=512^{2}\right)$ degraded by a Gaussian blur of size $5 \times 5$ and Poisson noise with scaling parameter $\alpha=0.5$. The parameter $\chi$ of (75) and the parameters $\left(\tau_{k}\right)_{1 \leq k \leq K}$ of $(76)$ are selected so as to maximize the SNR. The matrix $F$ is a tight frame version of the dual-tree transform proposed in [15] using symmlet of length 6 applied over 3 resolution levels $(\nu=2$ and $K=2 N)$. The same discrete gradient matrices $H$ and $V$ as in [4] are used. We aim at comparing the PPXA and Douglas-Rachford algorithms in the image decomposition problem under consideration. In both algorithms we set $\lambda_{n} \equiv 1$.

In this context, setting $\omega_{1}=\omega_{2}=\omega_{3}=1 / 3$, PPXA assumes the following form. 


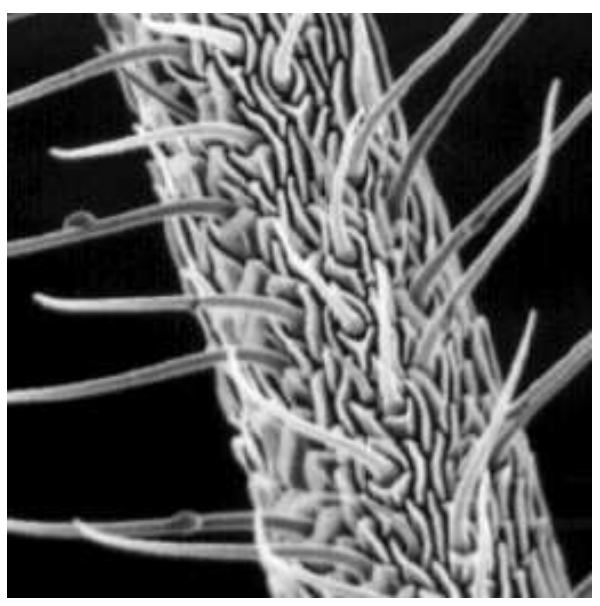

Original image $\bar{x}$

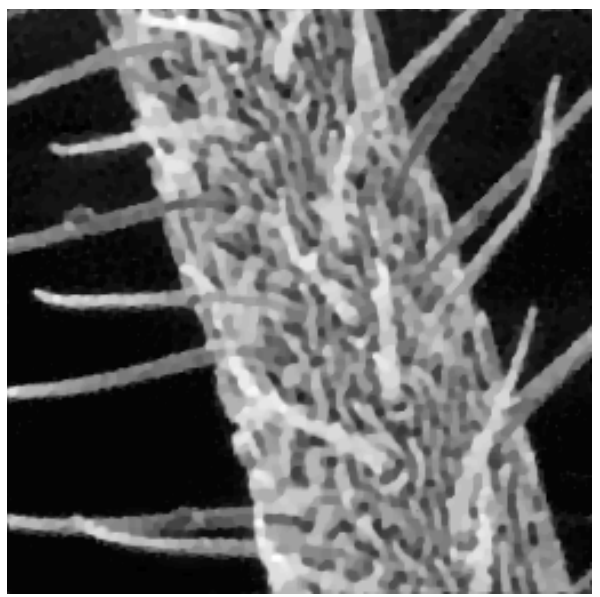

Geometry component $x_{1}$.

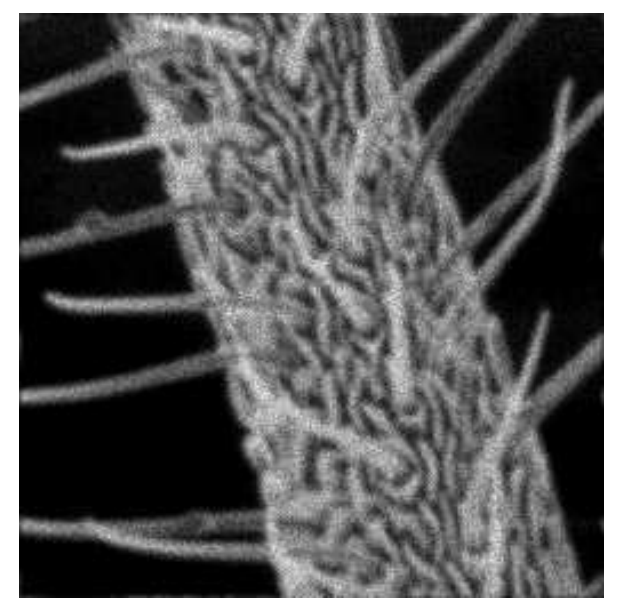

Degraded image $z$

$\mathrm{SNR}=14.2 \mathrm{~dB}-\mathrm{SSIM}=0.74$.

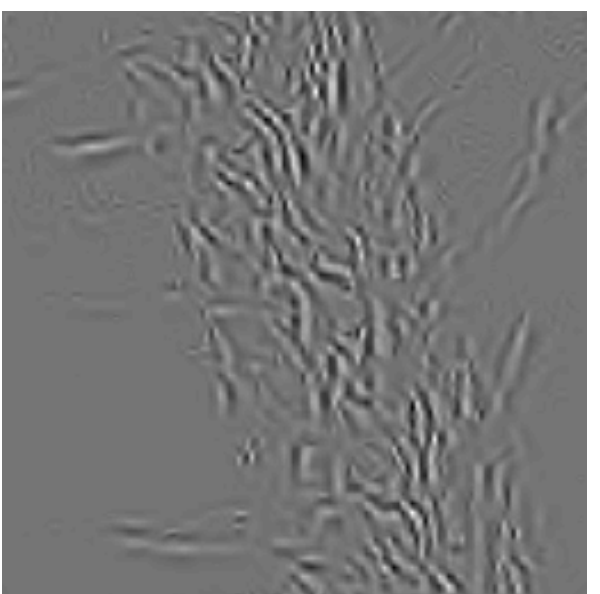

Texture component $F^{\top} x_{2}$.

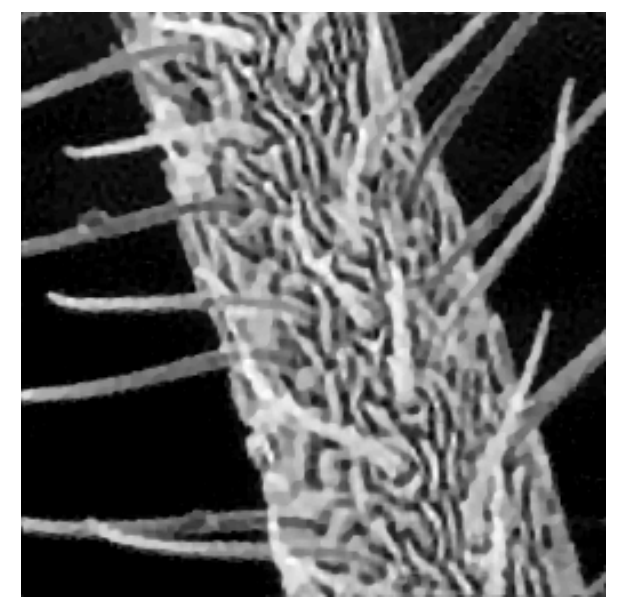

Restored image $x_{3}$

$\mathrm{SNR}=17.7 \mathrm{~dB}-\mathrm{SSIM}=0.86$.

Figure 5: Decomposition and restoration results. 
Initialization

$$
\mid \begin{aligned}
& \gamma=100 \\
& \left(y_{1,1,0}, \ldots, y_{6,1,0}\right)=\left(z, F^{\top} z, z, z, z, z\right) \\
& \left(y_{1,2,0}, \ldots, y_{6,2,0}\right)=\left(z, F^{\top} z, z, z, z, z\right) \\
& \left(y_{1,3,0}, \ldots, y_{6,3,0}\right)=\left(z, F^{\top} z, z, z, z, z\right) \\
& \text { For } i=1, \ldots, 6 \\
& \left\lfloor x_{i, 0}=\left(y_{i, 1,0}+y_{i, 2,0}+y_{i, 3,0}\right) / 3\right.
\end{aligned}
$$

For $n=0,1, \ldots$.

$$
\begin{aligned}
& u_{1,1, n}=y_{1,1, n} \\
& u_{2,1, n}=\operatorname{prox}_{3 \gamma h}\left(y_{2,1, n}\right) \\
& u_{3,1, n}=P_{C_{1}}\left(y_{3,1, n}\right) \\
& u_{4,1, n}=\operatorname{prox}_{3 \gamma g}\left(y_{4,1, n}\right) \\
& \left(u_{5,1, n}, u_{6,1, n}\right)=\operatorname{prox}_{3 \gamma \psi}\left(y_{5,1, n}, y_{6,1, n}\right) \\
& \left(u_{1,2, n}, u_{2,2, n}\right)=\left(y_{1,2, n}, y_{2,2, n}\right) \\
& u_{3,2, n}=P_{C_{2}}\left(y_{3,2, n}\right) \\
& \left(u_{4,2, n}, u_{5,2, n}, u_{6,2, n}\right)=\left(y_{4,2, n}, y_{5,2, n}, y_{6,2, n}\right) \\
& \left(u_{1,3, n}, \ldots, u_{6,3, n}\right)=P_{\operatorname{ker} \boldsymbol{L}_{1}}\left(y_{1,3, n}, \ldots, y_{6,3, n}\right) \\
& \text { For } i=1, \ldots, 6 \\
& \mid \begin{array}{l}
s_{i, n}=(1 / 3) \sum_{k=1}^{3} u_{i, k, n} \\
y_{i, 1, n+1}=y_{i, 1, n}+2 s_{i, n}-x_{i, n}-u_{i, 1, n} \\
y_{i, 2, n+1}=y_{i, 2, n}+2 s_{i, n}-x_{i, n}-u_{i, 2, n} \\
y_{i, 3, n+1}=y_{i, 3, n}+2 s_{i, n}-x_{i, n}-u_{i, 3, n} \\
x_{i, n+1}=x_{i, n}+s_{i, n}-x_{i, n}
\end{array}
\end{aligned}
$$


On the other hand, the Douglas-Rachford algorithm reduces to the following.

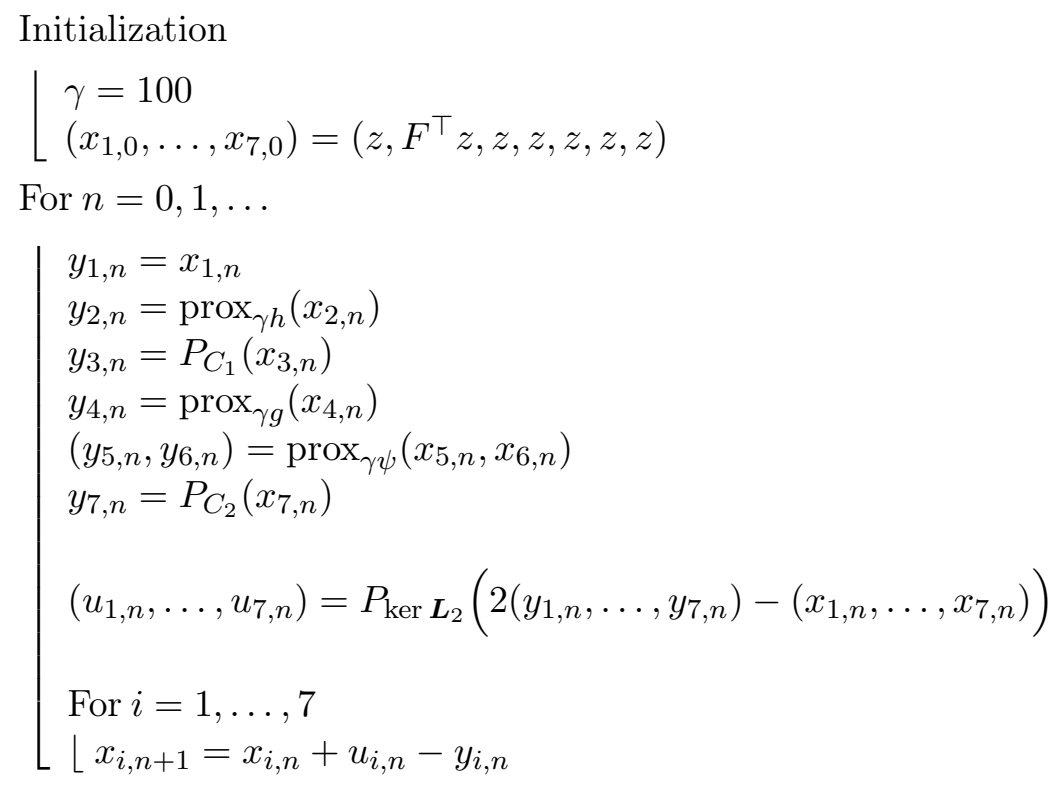

In Figure 6, the value of $\left\|\boldsymbol{y}_{n}-\boldsymbol{y}_{\infty}\right\| /\left\|\boldsymbol{y}_{0}-\boldsymbol{y}_{\infty}\right\|$ for the sequence $\left(\boldsymbol{y}_{n}\right)_{n \in \mathbb{N}}=\left(\left(y_{1, n}, \ldots, y_{7, n}\right)\right)_{n \in \mathbb{N}}$ of Theorem 4.4 and $\left\|\boldsymbol{x}_{n}-\boldsymbol{x}_{\infty}\right\| /\left\|\boldsymbol{x}_{0}-\boldsymbol{x}_{\infty}\right\|$ for the sequence $\left(\boldsymbol{x}_{n}\right)_{n \in \mathbb{N}}=\left(\left(x_{1, n}, \ldots, x_{6, n}\right)\right)_{n \in \mathbb{N}}$ of Theorem 4.7 (where $\boldsymbol{y}_{\infty}$ and $\boldsymbol{x}_{\infty}$ denote the respective limits) are plotted as a function of the computation time in seconds. In our experiments, 1000 iterations were used to produce a solution.

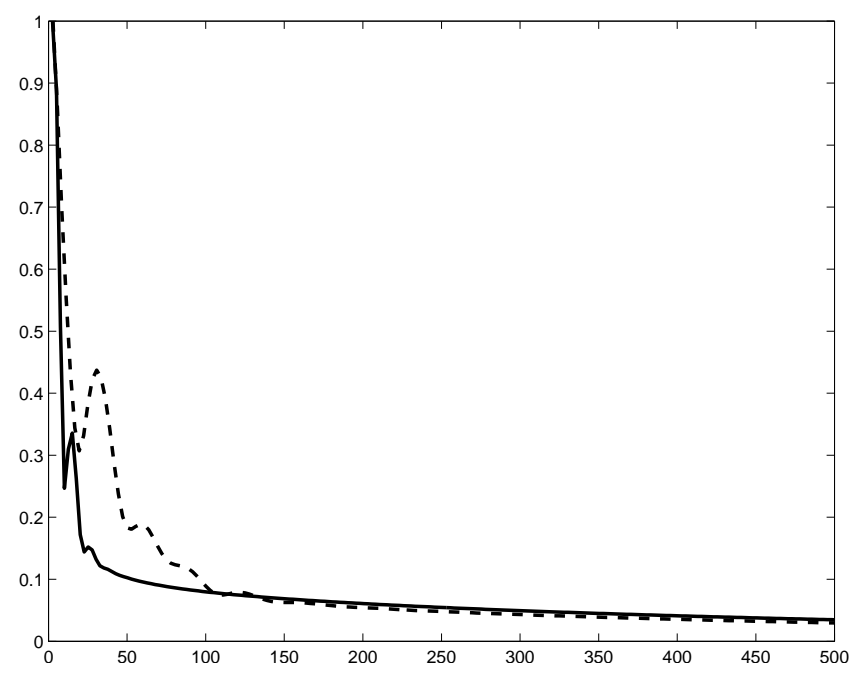

Figure 6: Convergence profiles of the Douglas-Rachford algorithm (solid line) and PPXA (dashed line) versus computation time in seconds. 


\section{Conclusion}

In this paper, the proximal formalism has been applied to multicomponent signal/image processing. Expressions of new proximity operators in product spaces have been derived. The proposed multicomponent framework has been illustrated through three different applications: stereocospy, multispectral imagery, and decomposition into geometry and texture components. Another field of application in which these techniques could be useful is the processing of color images. The proposed proximal formalism can also be used to derive algorithms for complex signal and image processing by regarding a complex signal as a signal with $m=2$ real components, namely its real and imaginary parts.

\section{References}

[1] Afonso, M.V., Bioucas-Dias, J.M., Figueiredo, M.A.T.: An augmented Lagrangian approach to the constrained optimization formulation of imaging inverse problems. IEEE Trans. Image Process. (2010). To appear.

[2] Anthoine, S., Pierpaoli, E., Daubechies, I.: Deux méthodes de déconvolution et séparation simultanées; application à la reconstruction des amas de galaxies. Traitement Signal 23, 439$447(2006)$

[3] Antoniadis, A., Fan, J.: Regularization of wavelet approximations. J. Amer. Statist. Assoc. 96, 939-967 (2001)

[4] Aujol, J.-F., Aubert, G., Blanc-Féraud, L., Chambolle, A.: Image decomposition into a bounded variation component and an oscillating component. J. Math. Imag. Vis. 22, 71-88 (2005)

[5] Aujol, J.-F., Chambolle, A.: Dual norms and image decomposition models. Int. J. Comput. Vis. 63, 85-104 (2005)

[6] Aujol, J.-F., Gilboa, G., Chan, T., Osher, S.J.: Structure-texture image decomposition Modeling, algorithms, and parameter selection. Int. J. Comput. Vis. 67, 111-136 (2006)

[7] Aujol, J.-F., Kang, S.H.: Color image decomposition and restoration. J. Vis. Comm. Image Represent. 17, 916-928 (2006)

[8] Bauschke, H. H. and Combettes, P. L.: Convex Analysis and Monotone Operator Theory in Hilbert Spaces. Springer-Verlag, New York. To appear.

[9] Beck, A., Teboulle, M.: A fast iterative shrinkage-thresholding algorithm for linear inverse problems. SIAM J. Imaging Sci. 2, 183-202 (2009)

[10] Briceño-Arias, L.M., Combettes, P.L.: Convex variational formulation with smooth coupling for multicomponent signal decomposition and recovery. Numer. Math. Theory Methods Appl. 2, 485-508 (2009) 
[11] Chambolle, A.: An algorithm for total variation minimization and applications. J. Math. Imag. Vis. 20, 89-97 (2004)

[12] Chan, T.F., Esedoglu, S., Park, F.E.: Image decomposition combining staircase reduction and texture extraction. J. Vis. Comm. Image Represent. 18, 464-486 (2007)

[13] Chaux, C., Benazza-Benyahia, A., Pesquet, J.-C., Duval, L.: Wavelet transform for the denoising of multivariate images. In: Collet, C., Chanussot, J., Chehdi, K. (eds.) Multivariate Image Processing, pp. 203-237. Wiley, New York (2010)

[14] Chaux, C., Combettes, P.L., Pesquet, J.-C., Wajs, V. R.: A variational formulation for framebased inverse problems. Inverse Probl. 23, 1495-1518 (2007)

[15] Chaux, C., Duval, L., Pesquet, J.-C.: Image analysis using a dual-tree $M$-band wavelet transform. IEEE Trans. Image Process. 15, 2397-2412 (2006)

[16] Combettes, P.L.: Iterative construction of the resolvent of a sum of maximal monotone operators. J. Convex Anal. 16, 727-748 (2009)

[17] Combettes, P.L., Pesquet, J.-C.: A Douglas-Rachford splitting approach to nonsmooth convex variational signal recovery. IEEE J. Selected Topics Signal Process. 1, 564-574 (2007)

[18] Combettes, P.L., Pesquet, J.-C.: Proximal thresholding algorithm for minimization over orthonormal bases. SIAM J. Optim. 18, 1351-1376 (2007)

[19] Combettes, P.L., Pesquet, J.-C.: A proximal decomposition method for solving convex variational inverse problems. Inverse Probl. 24, Article ID 065014 (2008)

[20] Combettes, P.L., Pesquet, J.-C.: Proximal splitting methods in signal processing. In: Bauschke et al. (eds.) Fixed-Point Algorithms for Inverse Problems in Science and Engineering. SpringerVerlag, New York (2010)

[21] Combettes, P.L., Wajs, V. R.: Signal recovery by proximal forward-backward splitting. Multiscale Model. Simul. 4, 1168-1200 (2005)

[22] Daubechies, I., Defrise, M., De Mol, C.: An iterative thresholding algorithm for linear inverse problems with a sparsity constraint. Comm. Pure Appl. Math. 57, 1413-1457 (2004)

[23] Daubechies, I., Teschke, G.: Variational image restoration by means of wavelets: simultaneous decomposition, deblurring and denoising. Appl. Comp. Harm. Anal. 19, 1-16 (2005)

[24] Deutsch, F.: Best Approximation in Inner Product Spaces. Springer-Verlag, New York (2001)

[25] Elad, M., Starck, J.-L., Donoho, D., Querre, P.: Simultaneous cartoon and texture image inpainting using morphological component analysis (MCA). Appl. Comp. Harm. Analysis 19, $340-358(2005)$

[26] Goldburg, M., Marks II, R.J.: Signal synthesis in the presence of an inconsistent set of constraints. IEEE Trans. Circ. Syst. 32, 647-663 (1985) 
[27] Huang, Y., Ng, M.K., Wen, Y.-W.: A fast total variation minimization method for image restoration. Multiscale Model. Simul. 7, 774-795 (2008)

[28] Hunt, B.R., Kübler, O.: Karhunen-Loève multispectral image restoration, part I: theory. IEEE Trans. Acoust. Speech Signal Process. 32, 592-600 (1984)

[29] Kang, M.: Generalized multichannel image deconvolution approach and its applications. Opt. Eng. 37, 2953-2964 (1998)

[30] Katsaggelos, A., Lay, K., Galatsanos, N.: A general framework for frequency domain multichannel signal processing. IEEE Trans. Image Process. 2, 417-420 (1993)

[31] Meyer, Y.: Oscillating Patterns in Image Processing and Nonlinear Evolution Equations. AMS, Providence, RI (2001)

[32] Miled, W., Pesquet, J.-C., Parent, M.: A convex optimization approach for depth estimation under illumination variation. IEEE Trans. Image Process. 18, 813-830 (2009)

[33] Moreau, J.-J.: Proximité et dualité dans un espace Hilbertien. Bull. Soc. Math. France 93, 273-299 (1965)

[34] Nesterov, Yu.: A method of solving a convex programming problem with convergence rate $O\left(1 / k^{2}\right)$. Soviet Math. Dokl. 27, 372-376 (1983)

[35] Nesterov, Yu.: Primal-dual subgradient methods for convex problems. Math. Program. 120, 221-259 (2009)

[36] Pedone, M., Heikkilä, J.: Blur and contrast invariant fast stereo matching. Lecture Notes in Comput. Sci. 5259, 883-890 (2008)

[37] Pustelnik, N., Chaux, C., Pesquet, J.C. Proximal methods for image restoration using a class of non-tight frame representations. Proc. Eur. Sig. and Image Proc. Conf. Aalborg, Danmark, Aug. 23-27 (2010)

[38] Rudin, L.I., Osher, S.J., Fatemi, E.: Nonlinear total variation based noise removal algorithms. Phys. D. 60, 259-268 (1992)

[39] Scharstein, D., Szeliski, R.: A taxonomy and evaluation of dense two-frame stereo correspondence algorithms. Int. J. Comput. Vis. 47, 7-42 (2002)

[40] Tschumperlé, D., Deriche, R.: Diffusion PDEs on vector-valued images. IEEE Signal Process. Mag. 19, 16-25 (2002)

[41] Vese, L.A., Osher, S.J.: Modeling textures with total variation minimization and oscillating patterns in image processing. J. Sci. Comput. 19, 553-572 (2003).

[42] Vese, L.A., Osher, S.J.: Image denoising and decomposition with total variation minimization and oscillatory functions. J. Math. Imag. Vis. 20, 7-18 (2004)

[43] Wang, Y., Yang, J., Yin, W., Zhang, Y.: A new alternating minimization algorithm for total variation image reconstruction. SIAM J. Imaging Sci. 1, 248-272 (2008) 
[44] Wang, Z., Bovik, A. C., Sheikh, H. R., Simoncelli, E.P.: Image quality assessment: from error visibility to structural similarity. IEEE Trans. Image Process. 13, 600-612 (2004)

[45] Weiss, P., Aubert, G., Blanc-Féraud, L.: Efficient schemes for total variation minimization under constraints in image processing. SIAM J. Sci. Comput. 31, 2047-2080 (2009)

[46] Wen, Y.-W., Ng, M.K., Ching, W.-K.: Iterative algorithms based on decoupling of deblurring and denoising for image restoration. SIAM J. Sci. Comput. 30, 2655-2674 (2008)

[47] Zălinescu, C.: Convex Analysis in General Vector Spaces. World Scientific, River Edge, NJ (2002) 